M. Maruyama

Nagoya Math. J.

Vol. 58 (1975), 25-68

\title{
STABLE VECTOR BUNDLES ON AN ALGEBRAIC SURFACE
}

\author{
MASAKI MARUYAMA*)
}

\section{Introduction.}

Let $X$ be a non-singular projective algebraic curve over an algebraically closed field $k$. D. Mumford introduced the notion of stable vector bundles on $X$ as follows;

Definition ([7]). A vector bundle $E$ on $X$ is stable if and only if for any non-trivial quotient bundle $F$ of $E$,

$$
\operatorname{deg}(E) / r(E)<\operatorname{deg}(F) / r(F),
$$

where $\operatorname{deg}(\cdot)$ denotes the degree of the first Chern class of a vector bundles and $r(\cdot)$ denotes the rank of a vector bundle.

D. Mumford, M. S. Narasimhan and C. S. Seshadri showed that the family of stable vector bundles on $X$ with given degree and rank has a coarse moduli scheme ([7], [11], [12], [13]). To prove this they used some special facts which were provided by the assumption that $X$ was a curve. For instance, (1) a coherent $\mathcal{O}_{X}$-module is torsion free if and only if it is locally free, (2) every vector bundle $E$ has a filtration $0=E_{0} \subset E_{1} \subset \cdots \subset E_{r-1} \subset E_{r}=E$ such that $E_{i} / E_{i-1}$ is a locally free $\mathcal{O}_{X}$-module of rank 1 , (3) the set of isomorphism classes of indecomposable vector bundles on $X$ with fixed degree (Chern class) and rank is bounded ${ }^{1)}$.

Let us consider higher dimensional cases. Assume that $X$ is a nonsingular projective variety over $k$ with $\operatorname{dim} X \geq 2$. Since, at least, the above three are not necessarily true, we have to overcome various difficulties to construct moduli of vector bundles on $X$. It is inevitable

Received January 9, 1974.

Revised December 19, 1974.

*) Partially supported by NSF grant GP 32969.

1) Let $X$ be an algebraic scheme over an algebraically closed field $k$. We say a set $S$ of coherent $\mathcal{O}_{X}$-modules is bounded if there exist an algebraic $k$-scheme $T$ and a $T$-flat coherent $\mathcal{O}_{X \times k T}$-module $F$ such that every member of $S$ is isomorphic to one of $\left\{F_{t}=F \otimes_{\mathcal{O}_{X \times T}} k(t) \mid t \in T(k)\right\}$. 
that, as the curve case, we have to restrict ourselves to a subfamily of vector bundles which satisfy some suitable conditions. Then what kind of properties should the conditions possess? First of all the family of vector bundles satisfying the conditions with given Chern classes and rank should be bounded. This is essential unlike the curve case because (3) above is not necessarily true in this case. Secondarily the conditions should be open conditions, that is, if $T$ is an algebraic $k$-scheme and if $F$ is a vector bundle on $X \times{ }_{k} T$, then the set of $k$-rational points $t$ of $T$ such that $F_{t}$ satisfy the conditions forms that of $k$-rational points of an open subset of $T$. Finally the subfamily should behave nicely when one takes a quotient by some equivalence relation (cf. §4). Now it seems to the author that the following condition is a hopeful candidate to fulfill our requirement.

Definition (Mumford-Takemoto [15]). Let us fix an ample linebundle $H$ on $X$. A torsion free coherent $\mathcal{O}_{X}$-module $E$ is stable (or, semi-stable) (with respect to $H$ ) if and only if for any non-trivial, non-torsion, quotient coherent $\mathcal{O}_{X}$-module $F$ of $E$,

$$
d(E, H) / r(E)<d(F, H) / r(F) \quad \text { (or, } \leq \text {, resp.) },
$$

where $d(\cdot, H)$ is the degree of the first Chern class of a coherent $\mathcal{O}_{X^{-}}$ module with respect to $H$.

The main purpose of this article is to show that if $X$ is a surface, then the family of stable vector bundles of rank 2 on $X$ is a good one.

In $\S 1$ we shall introduce the notion of vector bundles of type $\alpha_{1}, \cdots, \alpha_{r-1}$. Though this notion itself contains some important geometric meaning, we use it only to prove the main result of $\S 2$. $\S 1$ is devoted to modifying the results in $\S 1$ of [15] about stable vector bundles. In $\S 2$ we shall show that the set of isomorphism classes of stable vector bundles on an algebraic surface with fixed Chern classes and rank is bounded. In rank 2 case this was proved by F. Takemoto [15] and D. Mumford (unpublished). Though the basic idea of our proof is the same as theirs, we need the notion of type $\alpha_{1}, \cdots, \alpha_{r-1}$ to prove it in every rank and by our method we get more general results. In fact the above result is a special case of our theorem (Theorem 2.5 and corollaries to it). Openness of the stable vector bundle will be proved in $\S 3$ (Corollary 3.4.1). In $\S 4$ we shall construct a coarse moduli scheme of the family of stable vector bundles of rank 2 on a non-singular 
projective surface (Theorem 4.10). We use the assumption rank 2 only in Lemma 4.1. If we can replace it by some suitable lemma, then the restriction rank 2 can be omitted (see Remark 4.12). Our method is essentially the same as what Seshadri used in his proof in the curve case. But he used some facts which are peculiar to a curve. In our case we have to analyze more deeply the action of the group PGL(N) on some special schemes. We shall discuss a little bit about singularities of the moduli in the final part of $\S 4$.

\section{Notation and convention.}

Throughout this paper $k$ denotes an algebraically closed field and all varieties are reduced and irreducible algebraic $k$-schemes. We use the terms "vector bundles" and "locally free sheaves" interchangeablly. Let $X$ be a non-singular projective variety over $k$. If $E$ is a coherent $\mathcal{O}_{X}$-module of rank $r$, then we can define the Chern classes $c_{1}(E), \cdots, c_{r}(E)$ of $E$ (see [1]). For a coherent $\mathcal{O}_{X}$-module $F, h^{i}(F)$ denotes $\operatorname{dim}_{k} H^{i}(X, F)$ and $\chi(F)$ denotes $\Sigma(-1)^{i} h^{i}(F)$. For a divisor $D$ on $X, \mathcal{O}_{X}(D)$ denotes the linebundle defined by $D$. If $L$ is a linebundle on $X$, then $|L|$ denotes the complete linear system $|D|$ for a divisor $D$ on $X$ with $\mathcal{O}_{X}(D) \cong L$. For $S$-schemes $Z$ and $T, Z(T)$ denotes the set of $T$-valued points of $Z$, that is, $Z(T)=\operatorname{Hom}_{S}(T, Z)$ and in particular if $Z$ is an algebraic $k$ scheme, then $Z(k)$ means the set of $k$-rational points of $Z$. For a scheme $S$ and a coherent $\mathcal{O}_{S}$-module $E, \mathbf{P}(E)$ denotes Proj $\left(S_{O_{S}}(E)\right)$, where $S_{O_{S}}(E)$ is the $\mathcal{O}_{S}$-symmetric algebra of $E$.

The main part of this work was done while the author stayed at Mathematics Department, Harvard University in the academic year 1972-73. He wishes to thank all the people who made it possible. He also wishes to thank Professors H. Hironaka and D. Mumford for their encouragement and valuable suggestions.

\section{\$1. Vector bundles of type $\alpha_{1}, \cdots, \alpha_{r-1}$.}

Let $X$ be a non-singular projective variety over $k$ and let us fix a very ample linebundle $H$ on $X$. For any coherent $\mathcal{O}_{X}$-module $E, d(E, H)$ denotes the degree of the first Chern class of $E$ with respect to $H$ and $r(E)$ denotes the rank of $E$, that is, the rank of $E(x)=E \otimes_{O_{X}} k(x)$ as a vector space over $k(x)$ with the generic point $x$ of $X$. Now let us extend the notion of stable vector bundles. 
DEFINITION. Let $\alpha_{1}, \cdots, \alpha_{r-1}$ be a sequence of $r-1$ rational numbers. A torsion free coherent $\mathcal{O}_{X}$-module $E$ of rank $r$ on $X$ is called of type $\alpha_{1}, \cdots, \alpha_{r-1}$ (with respect to $H$ ) if and only if for any non-trivial, nontorsion, quotient coherent $\mathcal{O}_{X}$-module $F$ of $E$, the following inequalities are satisfied;

$$
d(E, H) / r(E)-\alpha_{s} \leq d(F, H) / r(F),
$$

where $s=r(F)(1 \leq s \leq r-1)$.

Remark 1.1. i) $E$ is semi-stable if and only if it is of type $0, \ldots, 0$.

ii) Take a sequence of $r-1$ rational numbers $\alpha_{1}, \cdots, \alpha_{r-1}$ such that $-1 / r s<\alpha_{s}<0$. Then $E$ is stable if and only if it is of type $\alpha_{1}, \cdots, \alpha_{r-1}$.

iii) In the definition we may assume that $F$ is torsion free (see [15]).

Let us show some lemmas which will be used often later.

LEMMA 1.2. A torsion free coherent $\mathcal{O}_{X}$-module $E$ of rank $r$ on $X$ is of type $\alpha_{1}, \cdots, \alpha_{r-1}$ if and only if for any non-trivial coherent $\mathcal{O}_{X^{-}}$ submodule $G$ of $E$, the following inequalities are satisfied;

$$
d(E, H) / r(E)+s \alpha_{r-s} /(r-s) \geq d(G, H) / r(G),
$$

where $s=r(G)(1 \leq s \leq r-1)$.

Proof. Put $F=E / G$, then by the definition $E$ is of type $\alpha_{1}, \cdots, \alpha_{r-1}$ if and only if for any $G$, we get

$$
d(E, H) / r(E)-\alpha_{r-s} \leq d(F, H) / r\left(F^{\prime}\right)
$$

because $r(F)=r(E)-r(G)=r-s$. Since $d(E, H)=d(F, H)+d(G, H)$, the above inequalities are equivalent to those in our lemma. q.e.d.

LEMMA 1.3. Let $E$ be a torsion free $\mathcal{O}_{X}$-module of rank $r$ and let $L$ be a linebundle on $X$. Then $E$ is of type $\alpha_{1}, \cdots, \alpha_{r-1}$ if and only if so is $E \otimes_{O_{X}} L$.

Proof. If one notes that the equality $c_{1}\left(F \otimes_{O_{X}} L\right)=c_{1}(F)+r(F) c_{1}(L)$ holds for any coherent $\mathcal{O}_{X}$-module $F$, the proof is obvious.

LEMMA 1.4. Let $E_{1}, E_{2}$ be two torsion free coherent $\mathcal{O}_{X}$-modules of rank $r$. Assume that there is an open subset $U$ of $X$ with $\operatorname{codim}(X-U, X)$ $\geq 2$ and an isomorphism $f: E_{1}\left|U \stackrel{\sim}{\longrightarrow} E_{2}\right| U$. Then $E_{1}$ is of type $\alpha_{1}, \cdots, \alpha_{r-1}$ if and only if so is $E_{2}$. 
Proof. It is clear that we have only to prove "if" part. Let $G_{1}$ be a coherent $\mathcal{O}_{X}$-submodule of $E_{1}$ of rank $s(1 \leq s \leq r-1)$. There is a coherent $\mathcal{O}_{X}$-submodule $G_{2}$ of $E_{2}$ such that $G_{2} \mid U=f\left(G_{1} \mid U\right)$ (E. G. A. Ch. I, 9.4.7). Since codim $(X-U, X) \geq 2$, we know that $c_{1}\left(E_{1}\right)=c_{1}\left(E_{2}\right)$ and $c_{1}\left(G_{1}\right)=c_{1}\left(G_{2}\right)$. On the other hand, $G_{2}$ satisfies the inequality in Lemma 1.2. Thus $G_{1}$ does it too, whence $E_{1}$ is of type $\alpha_{1}, \cdots, \alpha_{r-1}$.

q.e.d.

For a coherent $\mathcal{O}_{X}$-module $E$ of rank $r$, put $E^{\vee}=\mathscr{H}_{o_{o_{O}}}\left(E, \mathcal{O}_{X}\right)$, then $E^{\vee}$ is a torsion free coherent $\mathcal{O}_{X}$-module of rank $r$.

LEMMA 1.5. Let $E$ be a torsion free coherent $\mathcal{O}_{X}$-module of rank $r$. Then $E$ is of type $\alpha_{1}, \cdots, \alpha_{r-1}$ if and only if $E^{\vee}$ is of type $\alpha_{r-1} /(r-1)$, $\cdots, s \alpha_{r-s} /(r-s), \cdots,(r-1) \alpha_{1}$.

Proof. Let $F^{*}$ be a torsion free, quotient coherent $\mathcal{O}_{X}$-module of $E$ of rank $s(1 \leq s \leq r-1)$. There exists an open subset $U$ of $X$ such that $\operatorname{codim}(X-U, X) \geq 2$ and that $E \mid U$ is locally free. Then $\left(E^{\vee}\right)^{\vee} \mid U$ $=E \mid U$. On the other hand, there are natural inclusion $i: E \rightarrow\left(E^{\vee}\right)^{\vee}$ and $j: F=\mathscr{H}_{o m_{\mathcal{O}_{X}}}\left(F^{*}, \mathcal{O}_{X}\right) \rightarrow\left(E^{\vee}\right)^{\vee}$. If we put $F^{\prime}=i^{-1}(j(F))$, then we know that $F^{\prime}|U \cong F| U$. Thus we get that $c_{1}\left(F^{\prime}\right)=c_{1}(F)=-c_{1}\left(F^{*}\right)$ because $F^{*}$ is torsion free and $\operatorname{codim}(X-U, X) \geq 2$. Now assume that $E$ is of type $\alpha_{1}, \cdots, \alpha_{r-1}$. Then by virtue of Lemma 1.2 the following inequality holds ;

$$
d\left(F^{\prime}, H\right) / r\left(F^{\prime}\right) \leq d(E, H) / r(E)+s \alpha_{r-s} /(r-s) .
$$

Clearly this is equivalent to the following;

$$
d\left(E^{\vee}, H\right) / r\left(E^{\vee}\right)-s \alpha_{r-s} /(r-s) \leq d\left(F^{*}, H\right) / r\left(F^{*}\right) .
$$

We know therefore that $E^{\vee}$ is of type $\alpha_{r-1} /(r-1), \cdots, s \alpha_{r-s} /(r-s)$, $\cdots,(r-1) \alpha_{1}$ (see Remark 1.1, (iii)). If we replace $E$ by $E^{\vee}$ in the above argument, then we get the converse by virtue of Lemma 1.4 because $E\left|U \cong\left(E^{\vee}\right)^{\vee}\right| U$.

q.e.d.

LEMMA 1.6. Let $E$ be the same as in Lemma 1.5. Then $E$ is of type $\alpha_{1}, \cdots, \alpha_{r-1}$ with respect to $H$ if and only if it is of type $n \alpha_{1}, \cdots, n \alpha_{r-1}$ with respect to $n H$.

Proof. Obvious.

The following lemma is a key in the next section. 
LEMMA 1.7. If a torsion free $\mathcal{O}_{X}$-module $E$ of rank $r$ is of type $\alpha_{1}, \cdots, \alpha_{r-1}$ and if $d(E, H)<-r \alpha_{r-1} /(r-1)$, then $H^{\circ}(X, E)=0$.

Proof. Assume that $H^{0}(X, E) \neq 0$ and pick a non-zero element $s$ of $H^{0}(X, E)$. By the multiplication of $s$ we get an inclusion $j: \mathcal{O}_{X} \rightarrow E$. We know that $d\left(j\left(\mathcal{O}_{X}\right), H\right)=0$. On the other hand, we have that $d\left(j\left(\mathcal{O}_{X}\right), H\right) \leq d(E, H) / r+\alpha_{r-1} /(r-1)<0$ by virtue of Lemma 1.2 and our assumption. This is a contradiction. Therefore we get that $H^{0}(X, E)$ $=0$.

q.e.d.

\section{§ 2. Boundedness of stable vector bundles on an algebraic surface.}

Our main aim in this section is to prove the boundedness of stable vector bundles with fixed Chern classes and rank on an algebraic surface. In the sequel we assume that $X$ is a non-singular projective surface. We also fix a very ample linebundle $H$ and if we say that a vector bundle or a torsion free $\mathcal{O}_{X}$-module is of type $\alpha_{1}, \cdots, \alpha_{r-1}$ or (semi-)stable, then it means that it is so with respect to $H$ unless otherwise stated. We shall use the notation $E(n)$ instead of $E \otimes_{{ }_{0 X}} H^{\otimes n}$. We denote $d(H, H)$ by $h$. Let $K_{X}$ be the canonical bundle of $X$. If $E$ is a torsion free $\mathcal{O}_{X}$-module, then there are only a finite number of points $x_{1}, \cdots, x_{n}$ such that the rank of $E \otimes_{\mathcal{O}_{X}} k\left(x_{i}\right)$ is greater than that of $E$. We call $x_{1}, \cdots, x_{n}$ pinch points of $E . \quad E$ is locally free on $X-\left\{x_{1}, \cdots, x_{n}\right\}$.

Let $E$ be a coherent $\mathcal{O}_{X}$-module of rank $r$ with Chern classes $c_{1}(E), c_{2}(E)$. Then we get the following formulae;

$$
\begin{gathered}
c_{1}(E(n))=c_{1}(E)+r n c_{1}(H), \\
c_{2}(E(n))=r(r-1) n^{2} h / 2+(r-1) n d(E, H)+c_{2}(E) .
\end{gathered}
$$

Thus Riemann-Roch theorem implies

$$
\begin{aligned}
\chi(E(n))= & r n^{2} h / 2+\left(2 d(E, H)-r d\left(K_{X}, H\right)\right) n / 2-\left(c_{1}(E), K_{X}\right) / 2 \\
& +\left(c_{1}(E), c_{1}(E)\right) / 2-c_{2}(E)+r \chi\left(\mathcal{O}_{X}\right) .
\end{aligned}
$$

Let $S^{r}\left(\alpha_{1}, \cdots, \alpha_{r-1} ; c_{1}, c_{2}\right)$ be the set of isomorphism classes of torsion free $\mathcal{O}_{X}$-modules of type $\alpha_{1}, \cdots, \alpha_{r-1}$ with Chern classes $c_{1}, c_{2}$ (modulo numerical equivalence).

LEMMA 2.1. There exists an integer $n_{0}$ such that for any $E \in S^{r}\left(\alpha_{1}\right.$, $\left.\cdots, \alpha_{r-1} ; c_{1}, c_{2}\right)$, and for any integer $n \geq n_{0}$, we get that $H^{0}(X, E(n)) \neq 0$. 
Proof. By the formula (2.3) there exists an integer $n_{1}$ such that $\chi(E(n))>0$ for any integer $n \geq n_{1}$. On the other hand, by Serre duality $h^{2}(E(n))=h^{0}\left(\operatorname{Hom}_{\mathcal{O}_{X}}\left(E(n), K_{X}\right)\right)$. Since $d\left(E(n)^{\vee} \otimes_{\mathcal{O}_{X}} K_{X}, H\right)=-d(E, H)-r n h$ $+d\left(K_{X}, H\right)$, there exists an integer $n_{2}$ such that $d\left(E(n)^{\vee} \otimes_{\mathcal{O}_{X}} K_{X}, H\right)<-r \alpha_{1}$ for any $n \geq n_{2}$. Moreover, $\operatorname{Hom}_{o_{X}}\left(E(n), K_{X}\right)$ is of type $\alpha_{r-1} /(r-1), \cdots$, $s \alpha_{r-s} /(r-s), \cdots,(r-1) \alpha_{1}$ by virtue of Lemma 1.3 and Lemma 1.5 because $\operatorname{Hom}_{\mathscr{O}_{X}}\left(E(n), K_{X}\right) \cong E(n)^{\vee} \otimes_{\mathscr{O}_{X}} K_{X}$. Thus if $n \geq n_{2}$, then $h^{2}(E(n))=0$ by virtue of Lemma 1.7. Now put $n_{0}=\max \left(n_{1}, n_{2}\right)$, then $0<\chi(E(n))$ $=h^{0}(E(n))-h^{1}(E(n)) \leq h^{0}(E(n))$.

The preceding lemma and the following are special facts in the case of a surface and they are fundamental tools for the induction process in the proof of our main theorem.

LEMMA 2.2. If $E$ is a torsion free $\mathcal{O}_{X}$-module, then there exist a unique vector bundle $E^{\prime}$ and an injective homomorphism $f: E \rightarrow E^{\prime}$ such that $f$ induces an isomorphism on $U$, where $X-U$ is the set of pinch points of $E$.

Proof. Let $\left\{x_{1}, \cdots, x_{m}\right\}$ be the set of pinch points of $E$. If the set is empty, then there is nothing to prove. Put $U=X-\left\{x_{1}, \cdots, x_{m}\right\}$ and let $i: U \rightarrow X$ be the inclusion. If there exist $E^{\prime}$ and $f$, then $E^{\prime} \cong i_{*} i^{*}(E)$ and $f$ is defined by the natural homomorphism $E \rightarrow i_{*} i^{*}(E)$, whence they are unique. Let us prove that $i_{*} i^{*}(E)$ is locally free $\mathcal{O}_{X}$-module. If $Y_{j}$ $=\operatorname{Spec}\left(\mathcal{O}_{X, x_{j}}\right)$ and if $u_{j}: Y_{j} \rightarrow X$ is the natural morphism, then $u_{j}$ is flat and we get the following diagram;

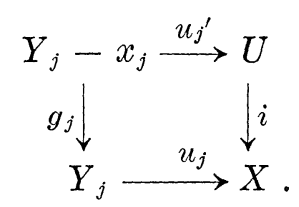

Since $i$ is of finite type and separated and since $u_{j}$ is flat, we have an isomorphism $u_{j}^{*} i_{*}\left(i^{*}(E)\right) \cong g_{j *}\left(u_{j}^{\prime}\right)^{*}\left(i^{*}(E)\right)$ (E. G. A., Ch. III, 1. 4. 15). Since $i_{*} i^{*}(E)$ is locally free if and only if $u_{j}^{*} i_{*}\left(i^{*}(E)\right)$ is free, we have only to prove that $g_{j *}\left(u_{j}{ }^{\prime}\right)^{*}\left(i^{*}(E)\right)$ is free. On the other hand, $\left(u_{j}{ }^{\prime}\right)^{*}\left(i^{*}(E)\right)$ is locally free. Thus we can reduce our assertion to Corollary 4.1.1 of [3]. Let $f$ be the natural morphism of $E$ to $i_{*} i^{*}(E)$. Then $\operatorname{Supp}(\operatorname{ker}(f))$ $\subset\left\{x_{1}, \cdots, x_{m}\right\}$. Hence $\operatorname{ker}(f)$ is a torsion $\mathcal{O}_{X}$-module. Since $E$ is torsion free, we know that $\operatorname{ker}(f)=0$.

q.e.d. 
Remark 2.3. i) As a matter of fact $E^{\prime}$ in the above lemma is isomorphic to $\left(E^{\vee}\right)^{\vee}$ and $f$ is the natural inclusion $E \rightarrow\left(E^{\vee}\right)^{\vee}$.

ii) Lemma 2.2 is not necessarily true if $\operatorname{dim} X$ is greater than 2.

LEMMA 2.4. Let $A$ be a noetherian integral domain such that for any $\mathfrak{p} \in \operatorname{Spec}(A), A_{\mathfrak{p}}$ is a U.F.D. and let $m$ be an element of a finitely generated torsion free $A$-module $M$. Then the following are equivalent to each other;

i) $M / A m$ is a torsion free A-module.

ii) For any $\mathfrak{p} \in \operatorname{Spec}(A)$ with $\mathrm{ht}(\mathfrak{p})=1$, if $m=a m^{\prime}$ for some $a \in A_{\mathfrak{p}}$, $m^{\prime} \in M$, then $a$ is a unit in $A_{p}$.

Proof. (ii) $\Rightarrow$ (i): First of all note that a finitely generated $A$-module $N$ is torsion free if and only if so is $N \otimes_{A} A_{\mathfrak{p}}$ for any maximal ideal $\mathfrak{p}$ of $A$. Thus replacing $A$ by $A_{\mathfrak{p}}$, we may assume that $A$ is a U. F. D.. Assume that $M / A m$ is not torsion free, then there are an element $m^{\prime}$ in $M$ and a non-unit element $a$ in $A$ such that $m^{\prime}$ is not contained in $A m$ and $a m^{\prime}=b m$ for some $b \in A$. We may assume that $a$ and $b$ contain no common divisors. If $a$ is a unit, then $m^{\prime}$ is contained in $A m$. Thus there is a prime ideal $\mathfrak{p}$ of $A$ such that $h t(\mathfrak{p})=1, a \in \mathfrak{p}$ and $b \notin \mathfrak{p}$. Then this is a contradiction because $a / b$ is not a unit in $A_{\mathfrak{p}}$ and $m=(a / b) m^{\prime}$.

(i) $\Rightarrow$ (ii): Assume that there are $\mathfrak{p} \in \operatorname{Spec}(A)$ with ht $(\mathfrak{p})=1, m^{\prime} \in M$ and a non-unit element $a$ in $A_{\mathfrak{p}}$ such that $m=a m^{\prime}$. If $m^{\prime}$ is contained in $m A_{p}$, then $m^{\prime}=a^{\prime} m=a a^{\prime} m^{\prime}$ for some $a^{\prime} \in A_{\mathfrak{p}}$, that is, $a a^{\prime}=1$ in $A_{\mathrm{p}}$ because $M$ is torsion free and $A_{\mathfrak{p}}$ is an integral domain. Hence $m^{\prime} \not \equiv 0 \bmod . A_{\mathfrak{p}} m$ and $a m^{\prime}=0 \bmod . A_{p} m$, which means that $M / A m$ is not torsion free. This is a contradiction.

q.e.d.

Now we come to the main theorem in this section.

THEOREM 2.5. Let $a$ be an integer and let $S_{a}^{r}\left(\alpha_{1}, \cdots, \alpha_{r-1} ; c_{1}\right)$ be the set $\coprod_{a \geq c_{2}} S^{r}\left(\alpha_{1}, \cdots, \alpha_{r-1} ; c_{1}, c_{2}\right)$. Then there are two constants $b_{0}, b_{1}$ (independent of each $\left.c_{2}\right)$ such that for any $E \in S_{a}^{r}\left(\alpha_{1}, \cdots, \alpha_{r-1} ; c_{1}\right), h^{0}(E) \leq b_{0}$ and $h^{0}\left(E \otimes_{O_{X}} \mathcal{O}_{C}\right) \leq b_{1}$ for any curve $C$ in an open set $U(E)$ of $|H|$, where $U(E)$ may depend on $E$.

Proof. A) Put $V S_{a}^{r}\left(\alpha_{1}, \cdots, \alpha_{r-1} ; c_{1}\right)=\left\{E \in S_{a}^{r}\left(\alpha_{1}, \cdots, \alpha_{r-1} ; c_{1}\right) \mid E\right.$ is locally free\}. First of all let us show that if the theorem is true for $V S_{a}^{r}\left(\alpha_{1}, \cdots, \alpha_{r-1} ; c_{1}\right)$, then so is for $S_{a}^{r}\left(\alpha_{1}, \cdots, \alpha_{r-1} ; c_{1}\right)$. For any 
$E \in S_{a}^{r}\left(\alpha_{1}, \cdots, \alpha_{r-1} ; c_{1}\right)$, there exists the following exact sequence by virtue of Lemma 2.2 ;

$$
0 \longrightarrow E \longrightarrow E^{\prime} \longrightarrow T \longrightarrow 0,
$$

where $E^{\prime}$ is locally free and $\operatorname{dim} \operatorname{Supp}(T) \leq 0$. It is clear that $c_{1}(E)=c_{1}\left(E^{\prime}\right)$. We claim that $c_{1}(T)=0$ and $c_{2}(T)=-h^{0}(T) \leq 0$. In fact, since $\operatorname{dim} \operatorname{Supp}(T)$ $\leq 0$ and $\operatorname{dim} X=2$, we know that $c_{1}(T)=0$ and $h^{1}(T)=h^{2}(T)=0$. This and Riemann-Roch theorem imply that $h^{0}(T)=\chi(T)=-c_{2}(T)$. Thus $c_{2}\left(E^{\prime}\right)=c_{2}(E)-h^{0}(T) \leq a$. On the other hand, by virtue of Lemma 1.4 $E^{\prime}$ is of type $\alpha_{1}, \cdots, \alpha_{r-1}$. We know therefore that $E^{\prime}$ is contained in $V S_{a}^{r}\left(\alpha_{1}, \cdots, \alpha_{r-1} ; c_{1}\right)$. By the assumption that our theorem is true for $V S_{a}^{r}\left(\alpha_{1}, \cdots, \alpha_{r-1} ; c_{1}\right)$ we get $b_{0}, b_{1}, U\left(E^{\prime}\right)$ such that $h^{0}\left(E^{\prime}\right) \leq b_{0}, h^{0}\left(E^{\prime} \otimes_{\mathcal{O}_{X}} \mathcal{O}_{C}\right)$ $\leq b_{1}$ for any $C \in U\left(E^{\prime}\right)$. Since $h^{0}(E) \leq h^{0}\left(E^{\prime}\right)$ and since $h^{0}\left(E \otimes_{O_{X}} \mathcal{O}_{C}\right)$ $=h^{0}\left(E^{\prime} \otimes_{O_{X}} \mathcal{O}_{C}\right)$ if $C$ goes through none of pinch points of $E, b_{0}, b_{1}$ and $U(E)=\left\{C \in U\left(E^{\prime}\right) \mid C\right.$ goes through none of pinch points of $\left.E\right\}$ are the desired ones.

B) Let $S^{1}\left(c_{1}, c_{2}\right)$ be the set of isomorphism classes of torsion free $\mathcal{O}_{X}$-modules of rank 1 with Chern classes $c_{1}, c_{2}$. Put $S_{a}^{1}\left(c_{1}\right)=\coprod_{a \geq c_{2}} S^{1}\left(c_{1}, c_{2}\right)$. Let us prove that for $S_{a}^{1}\left(c_{1}\right)$, there exist constants $b_{0}, b_{1}$ which satisfy the consequence of our theorem. Let $L\left(c_{1}\right)$ be the set of isomorphism classes of invertible $\mathcal{O}_{X}$-modules with Chern class $c_{1}$. Then $L\left(c_{1}\right)$ is bounded because it is parametrized by a finite number of connected components of Pic $(X)$. Thus there is a constant $b_{0}$ such that $h^{0}(L) \leq b_{0}$ for any $L \in L\left(c_{1}\right)$. Let $C$ be a non-singular curve in $|H|$. Then $h^{0}\left(L \otimes_{\mathcal{O}_{X}} \mathcal{O}_{C}\right)$ $\leq \max \left\{d\left(c_{1}, H\right)-\left(h+d\left(K_{X}, H\right)\right) / 2-1, d\left(c_{1}, H\right) / 2,0\right\}=b_{1}$ by Riemann-Roch theorem and Clifford's theorem. Thus by the same argument in (A) we know that the $b_{0}, b_{1}$ above are the desired constants.

C) Assume that the theorem is true in the case of rank $r-1$. Under this assumption we shall show that for $V S_{a}^{r}\left(\alpha_{1}, \cdots, \alpha_{r-1} ; c_{1}\right)$ our theorem holds. For any $E \in V S_{a}^{r}\left(\alpha_{1}, \cdots, \alpha_{r-1} ; c_{1}\right)$ we get the following by virtue of the formula (2.3);

$$
\begin{aligned}
\chi(E(n))= & r n^{2} h / 2+\left(2 d(E, H)-r d\left(K_{X}, H\right)\right) n / 2 \\
& +\left(c_{1}(E), c_{1}(E)-K_{X}\right) / 2-c_{2}(E)+r \chi\left(\mathcal{O}_{X}\right) \\
\geq & r n^{2} h / 2+\left(2 d\left(c_{1}, H\right)-r d\left(K_{X}, H\right)\right) n / 2 \\
& +\left(c_{1}, c_{1}-K_{X}\right) / 2-a+r \chi\left(\mathcal{O}_{X}\right) .
\end{aligned}
$$

If $n$ is sufficiently large, then the right hand side of the above is positive, 
whence so is the left hand side. Thus by a similar argument in the proof of Lemma 2.1 we know that there is an integer $n_{0}$ such that $h^{0}(E(n))>0$ for any $n \geq n_{0}$ and any $E \in V S_{a}^{r}\left(\alpha_{1}, \cdots, \alpha_{r-1} ; c_{1}\right)$. Let us fix an integer $n \geq n_{0}$ and take an element $E$ of $V S_{a}^{r}\left(\alpha_{1}, \cdots, \alpha_{r-1} ; c_{1}\right)$. Pick a non-zero element $s$ of $H^{0}(X, E(n))$. Assume that there is a positive divisor $D$ such that $s$ can be written in the form of $s=s_{1} \otimes s_{2}$ with $s_{1} \in H^{0}\left(X, E(n) \bigotimes_{\mathcal{O}_{X}} \mathcal{O}_{X}(-D)\right), s_{2} \in H^{0}\left(X, \mathcal{O}_{X}(D)\right)$. Then there is a maximal element $D$ in the set of such divisors because the degrees of them are bounded above. For the maximal element $D$, if $s_{1, x}=u t_{x}$ with some $u \in \mathcal{O}_{X, x}, t \in\left(E(n) \otimes_{\mathcal{O}_{X}} \mathcal{O}_{X}(-D)\right)_{x}$, then $u$ is a unit in $\mathcal{O}_{X, x}$. Hence in the following exact sequence $F^{\prime}$ is torsion free by virtue of Lemma 2.3;

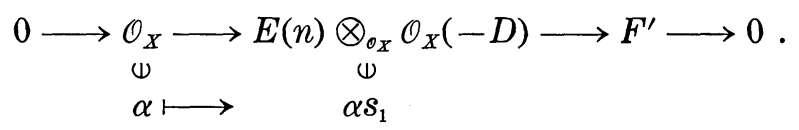

This sequence yields the following exact sequence;

$$
0 \longrightarrow \mathcal{O}_{X}(D) \otimes_{O_{X}} H^{\otimes-n} \longrightarrow E \longrightarrow F \longrightarrow 0,
$$

where $F$ is torsion free. Put $\mathscr{L}=\{D \mid D$ is obtained as above from some $\left.E \in V S_{a}^{r}\left(\alpha_{1}, \cdots, \alpha_{r-1} ; c_{1}\right)\right\} /$ (linear equivalence) and $\mathscr{N}=\mathscr{L} /$ (numerical equivalence). Then we claime that $\sharp(\mathscr{N})<\infty$. In fact since $\mathcal{O}_{X}(D)$ is an $\mathcal{O}_{X^{-}}$ submodule of $E(n)$ for some locally free $\mathcal{O}_{X}$-module $E$ of type $\alpha_{1}, \cdots, \alpha_{r-1}$ with $c_{1}(E)=c_{1}$, we have by virtue of Lemma 1.2 that $d(D, H) \leq$ $d(E(n), H) / r(E(n))+\alpha_{r-1} /(r-1)=d\left(c_{1}, H\right) / r+n h+\alpha_{r-1} /(r-1)$. Moreover $D$ is a positive divisor. Hence $\sharp(\mathscr{N})<\infty$ (see p. 113 of [9]). On the other hand, it is clear that $c_{1}(F)=c_{1}(E)-D+n c_{1}(H)$. We get therefore that $c_{2}(F)=c_{2}(E)-\left(c_{1}(F), c_{1}\left(\mathcal{O}_{X}(D) \otimes_{\mathcal{O}_{X}} H^{\otimes-n}\right)\right)=c_{2}(E)-\left(c_{1}-D\right.$ $\left.+n c_{1}(H), D-n c_{1}(H)\right)$. Since $\left(c_{1}-D+n c_{1}(H), D-n c_{1}(H)\right)$ depends only on the numerical equivalence class of $D$ and since $\sharp(\mathscr{N})<\infty,\left\{-\left(c_{1}-D\right.\right.$ $+n H, D-n H) \mid D \in \mathscr{L}\}$ is bounded above. Put $\beta=\max _{D \in \mathscr{L}}\left\{-\left(c_{1}-D+n H, D\right.\right.$ $-n H)\}$. Then $c_{2}(F) \leq a+\beta$ for any $F$ obtained as above. Let us prove that every $F$ obtained from some $E$ in $V S_{a}^{r}\left(\alpha_{1}, \cdots, \alpha_{r-1} ; c_{1}\right)$ is of type $\alpha_{1}{ }^{\prime}, \cdots, \alpha_{r-2}{ }^{\prime}$ for some sequence of $r-2$ rational numbers $\alpha_{1}{ }^{\prime}, \cdots, \alpha_{r-2}{ }^{\prime}$. Let $p: F \rightarrow G$ be a surjective homomorphism of $F$ to a coherent $\mathcal{O}_{X^{-}}$ module $G$ of ranks $s(1 \leq s \leq r-2)$. Since the natural homomorphism $q: E \longrightarrow F \stackrel{p}{\longrightarrow} G$ is surjective and since $E$ is of type $\alpha_{1}, \cdots, \alpha_{r-1}$, we obtain the following inequality; 


$$
d(E, H) / r-\alpha_{s} \leq d(G, H) / s
$$

Meanwhile,

$$
\begin{aligned}
d(E, H) / r-\alpha_{s}=d(E, H) /(r-1)- & d(E, H) / r(r-1)-\alpha_{s} \\
=d(F, H) /(r-1)- & {[1 /(r-1)\{n h-d(D, H)} \\
& \left.\left.+d\left(c_{1}, H\right) / r\right\}+\alpha_{s}\right] .
\end{aligned}
$$

If we put $\alpha_{s, D}=\left[1 /(r-1)\left\{n h-d(D, H)+d\left(c_{1}, H\right) / r\right\}+\alpha_{s}\right]$, then $\alpha_{s, D}$ depends only on the numerical equivalence class of $D$. Since $\#(\mathscr{N})<\infty$, $\alpha_{s, D}$ ranges over a finite number of rational numbers. Take the number $\alpha_{s}^{\prime}=\max _{D \in \mathscr{L}}\left\{\alpha_{s, D}\right\}$, then the above inequality implies

$$
d(F, H) /(r-1)-\alpha_{s}^{\prime} \leq d(G, H) / s .
$$

Hence $F$ is of type $\alpha_{1}^{\prime}, \cdots, \alpha_{r-2}{ }^{\prime}$. If $\mathscr{Q}$ is the set of isomorphism classes of $F^{\prime}$ 's which are obtained from some $E$ in $V S_{a}^{r}\left(\alpha_{1}, \cdots, \alpha_{r-1} ; c_{1}\right)$ as above, then the above results imply

$$
\mathscr{Q} \subset \coprod_{\lambda \in \mathcal{N}} \coprod_{a+\beta \geq c_{2}} S^{r-1}\left(\alpha_{1}{ }^{\prime}, \cdots, \alpha_{r-2}{ }^{\prime} ; c_{1}-\lambda+n \lambda_{H}, c_{2}\right),
$$

where $\lambda_{H}$ is the numerical equivalence class of $c_{1}(H)$. By the assumption that the theorem is true in the case of rank $r-1$, there are two constants $b_{0, \lambda}, b_{1, \lambda}$ satisfying the conditions of our theorem for $S_{a+\beta}^{r-1}\left(\alpha_{1}^{\prime}\right.$, $\left.\cdots, \alpha_{r-2}^{\prime} ; c_{1}-\lambda+n \lambda_{H}\right)$. Thus if we put $b_{i, 1}=\max _{\lambda \in \mathcal{N}}\left\{b_{i, \lambda}\right\} \quad(i=0,1)$, then for any $F \in \mathscr{Q}, h^{0}(F) \leq b_{0,1}$ and $h^{0}\left(F \otimes_{\mathcal{O}_{X}} \mathcal{O}_{C}\right) \leq b_{1,1}$ for $C \in U(F)$, where $U(F)$ is a suitable open set of $|H|$. On the other hand, there are two constants $b_{0,2}, b_{1,2}$ such that $h^{0}\left(\mathcal{O}_{X}(D) \otimes_{\mathcal{O}_{X}} H^{\otimes-n}\right) \leq b_{0,2}$ and $h^{0}\left(\mathcal{O}_{X}(D) \otimes_{\mathcal{O}_{X}} H^{\otimes-n}\right.$ $\left.\otimes_{O_{X}} \mathcal{O}_{C}\right) \leq b_{1,2}$ for any $D \in \mathscr{L}$, where $C$ is contained in a suitable open set $U$ of $|H|$, because $\sharp(\mathscr{N})<\infty$ (see the proof in (B)). Thus we get

$$
\begin{aligned}
h^{0}(E) & \leq h^{0}\left(\mathcal{O}_{X}(D) \otimes_{\mathcal{O X}_{X}} H^{\otimes-n}\right)+h^{0}(F) \\
& \leq b_{0,1}+b_{0,2}, \\
h^{0}\left(E \otimes_{\mathcal{O}_{X}} \mathcal{O}_{C}\right) & \leq h^{0}\left(\mathcal{O}_{X}(D) \otimes_{\mathcal{O}_{X}} H^{\otimes-n} \otimes_{\mathcal{O}_{X}} \mathcal{O}_{C}\right)+h^{0}\left(F \otimes_{\mathcal{O}_{X}} \mathcal{O}_{C}\right) \\
& \leq b_{1,1}+b_{1,2},
\end{aligned}
$$

where $C$ is contained in the subset $U(E)=\left\{C \in U\left(F^{\prime}\right) \cap U \mid C\right.$ goes through none of the pinch points of $F\}$. It is clear that $U(E)$ is open in $|H|$. Consequently we obtain $b_{0}=b_{0,1}+b_{0,2}, b_{1}=b_{1,1}+b_{1,2}$ and $U(E)$ which satisfy the conditions of the theorem. 
(D) If the theorem holds in the case of rank $r-1$, then we get the theorem for locally free $\mathcal{O}_{X}$-modules of rank $r$ by virtue of (C). Then (A) implies that the theorem holds in the case of rank $r$. Combining this and (B) we complete the proof of our theorem by induction on $r$.

COROLlaRY 2.5.1. $S^{r}\left(\alpha_{1}, \cdots, \alpha_{r-1} ; c_{1}, c_{2}\right)$ is bounded for every $\alpha_{1}, \cdots$, $\alpha_{r-1}, c_{1}, c_{2}$. In particular the set of (semi-) stable vector bundles with fixed Chern classes and rank on a non-singular projective surface is bounded.

Proof. It is obvious by virtue of our theorem and Theorem 1.13 of [4].

COROLlARY 2.5.2. The set of second Chern classes of torsion free $\mathcal{O}_{X}$-modules of type $\alpha_{1}, \cdots, \alpha_{r-1}$ with a fixed first Chern class $c_{1}$ (numerical equivalence) is bounded below.

Proof. Fix an integer $n$ such that $d\left(E(n)^{\vee} \otimes_{\mathcal{O}_{X}} K_{X}, H\right)=d\left(K_{X}, H\right)$ $-d\left(c_{1}, H\right)-n r h<-r \alpha_{1}$. Then $h^{2}(E(n))=0$ for any torsion free $\mathcal{O}_{X}$-module of type $\alpha_{1}, \cdots, \alpha_{r-1}$ with the first Chern class $c_{1}$ (see the proof of Lemma 2.1). Let us consider the set $S_{a}^{r}\left(\alpha_{1}, \cdots, \alpha_{r-1} ; c_{1}+n r \lambda_{H}\right)$ (the notation is the same as in the proof of Theorem 2.5). For every $F \in$ $S_{a}^{r}\left(\alpha_{1}, \cdots, \alpha_{r-1} ; c_{1}+n r \lambda_{H}\right)$, we get

$$
c_{2}(F(-n))=c_{2}(F)-r(r-1) h n^{2} / 2-(r-1) d\left(c_{1}, H\right) n .
$$

Theorem 2.5 implies that there is an constant $b_{0}$ such that $h^{0}(F) \leq b_{0}$. On the other hand, $h^{0}(F) \geq \chi(F)=r \chi\left(\mathcal{O}_{X}\right)+\left(c_{1}+n r c_{1}(H), c_{1}+n r c_{1}(H)\right.$ $\left.-K_{X}\right) / 2-c_{2}(F)$ by Riemann-Roch theorem because $F=E(n)$ with some torsion free $\mathcal{O}_{X}$-module $E$ of type $\alpha_{1}, \cdots, \alpha_{r-1}$ with the first Chern class $c_{1}$. Thus $b_{0} \geq h^{0}(F) \geq A-c_{2}(F)$ with some constant $A$, whence $\left\{c_{2}(F) \mid F\right.$ is a torsion free $\mathcal{O}_{X}$-module of type $\alpha_{1}, \cdots, \alpha_{r-1}$ with the first Chern class $\left.c_{1}\right\}$ is bounded below. Hence by virtue of the relation between the second Chern classes of $F$ and $F(-n)$ this implies our assertion q.e.d.

COROLlaRY 2.5.3. $S_{a}^{r}\left(\alpha_{1}, \cdots, \alpha_{r-1} ; c_{1}\right)$ in Theorem 2.5 is bounded for any $\alpha_{1}, \cdots, \alpha_{r-1}, c_{1}, a$.

CoRollary 2.5.4. For a vector bundle $E$ of rank $r$ on $X$, put $\Delta(E)=(r-1)\left(c_{1}(E), c_{1}(E)\right)-2 r c_{2}(E)\left(\Delta(E)=-c_{2}\left(\operatorname{End}_{o X}(E)\right)\right)$. There is a 
constant $C$ such that $\Delta(E)<C$ for any vector bundle $E$ of type $\alpha_{1}, \cdots, \alpha_{r-1}$ on $X$.

Proof. For any linebundle $L, \Delta(E)=\Delta\left(E \otimes_{0_{X}} L\right)$ and $\Delta(E)$ depends only on the numerical equivalence classes of $c_{1}(E), c_{2}(E)$. Thus we may assume that $c_{1}(E)$ ranges a finite number of numerical equivalence classes because $\operatorname{Pic}(X) / \operatorname{Pic}^{\tau}(X)$ is a finitely generated abelian group. Then by virtue of Corollary 2.5.2 the set of the numerical equivalence classes of $c_{2}(E)^{\prime}$ 's is bounded below, whence the set of $\Delta(E)$ 's is bounded above.

q.e.d.

\section{§ 3. Openness of stable vector bundles on an algebraic surface.}

In this section we shall show that if there is a family of vector bundles $F$ on $X$, that is, $F$ is a locally free $\mathcal{O}_{X \times T}$-module with some locally of finite type $k$-scheme $T$, then the set $S=\left\{t \in T(k) \mid F_{t}\right.$ is stable $\}$ is that of $k$-rational points of an open set of $T$.

LEMMA 3.1. Let $E$ be a torsion free coherent $\mathcal{O}_{X}$-module of rank $r$ generated by its global sections. Then there is a filtration $0=E_{0} \subset E_{1}$ $\subset \cdots \subset E_{r-1} \subset E_{r}=E$ such that $E_{i} / E_{i_{-1}} \cong \mathcal{O}_{X}$ for $1 \leq i \leq r-1$ and $E_{r} / E_{r-1}$ is a torsion free coherent $\mathcal{O}_{X}$-module of rank 1 .

Proof. Let $x_{1}, \cdots, x_{n}$ be the pinch points of $E$ and put $U=X$ $-\left\{x_{1}, \cdots, x_{n}\right\}$. If $\pi: Y=\mathbf{P}(E \mid U) \rightarrow U$ is the projective bundle associated with $E \mid U$, then we get a natural map $\theta: H^{0}(X, E) \rightarrow H^{0}(U, E \mid U)$ $=H^{0}\left(U, \pi_{*}\left(\mathcal{O}_{Y}(1)\right)\right)=H^{0}\left(Y, \mathcal{O}_{Y}(1)\right)$, where $\mathcal{O}_{Y}(1)$ is the tautological linebundle of $E \mid U$. Let $\mathscr{L}$ be the linear system defined by the image of $\theta$. We claim that $\mathscr{L}$ has no base points. In fact, if $y \in Y$ is a base point of $\mathscr{L}$ and $\pi(y)=x$, then $\left\{s(x) \mid s \in H^{0}(X, E)\right\}$ is a proper linear subspace of $E(x)$ because if one regards $s(x)$ as a linear form on $\mathbf{P}\left(\operatorname{Hom}_{k(x)}(E(x), k(x))\right)$, then $s(x)(y)=0$ for any $s \in H^{0}(X, E)$. This contradicts that $H^{0}(X, E)$ generates $E_{x}$. Then Bertini's theorem and the fact that $D_{x}(D \in \mathscr{L})$ is a hyperplane of $\mathbf{P}(E)_{x}$ for an $x \in X(k)$ imply that general members of $\mathscr{L}$ are irreducible. We know therefore that there is an element $s$ of $H^{0}(X, E)$ such that $F=\{x \mid s(x)=0\}$ is a closed set of $X$ with $\operatorname{codim}(F, X)$ $\geq 2$ because if codim $(F, X)=1$, then the divisor $D$ in $\mathscr{L}$ corresponding to $s$ is reducible. Such an $s$ gives rise to the following exact sequence;<smiles>O[14CH2][14CH]=[14C](O)[14CH2]O</smiles> 
where $E^{\prime}$ is a torsion free $\mathcal{O}_{X}$-module of rank $r-1$ by virtue of Lemma 2.3. Assume that there is a filtration $0=E_{0}{ }^{\prime} \subset E_{1}{ }^{\prime} \subset \cdots \subset E_{r-1}{ }^{\prime}=E^{\prime}$ such that $E_{i}{ }^{\prime} / E_{i_{-1}}{ }^{\prime} \cong \mathcal{O}_{X}, 1 \leq i \leq r-2$ and $E_{r-1}{ }^{\prime} / E_{r-2}{ }^{\prime}$ is torsion free. Put $E_{0}=0, E_{i}=p^{-1}\left(E_{i_{-1}}{ }^{\prime}\right)$, then we know that $E_{i} / E_{i_{-1}} \cong E_{i_{-1}}{ }^{\prime} / E_{i_{-2}}{ }^{\prime} \cong \mathcal{O}_{X}$ and $E_{r} / E_{r-1} \cong E_{r-1}^{\prime} / E_{r-2}{ }^{\prime}$ is torsion free. Thus we complete our proof by induction on $r$.

Corollary 3.1.1. If $E$ is as above, then $c_{1}(E) \geq 0^{2)}$ and $c_{2}(E) \geq 0$.

Proof. Take a filtration as in the above lemma. Then $c_{1}(E)$ $=c_{1}\left(E / E_{r-1}\right), c_{2}(E)=c_{2}\left(E / E_{r-1}\right)$ and $E / E_{r-1}$ is generated by its global sections because so is $E$. Thus we have only to prove our assertion in the case of rank 1. By virtue of Lemma 2.2 there is an imbedding of $E$ to a linebundle $L$ on $X$;

$$
0 \longrightarrow E \longrightarrow L \longrightarrow T \longrightarrow 0 \text {, }
$$

where $\operatorname{dim} \operatorname{Supp}(T) \leq 0$. Since $H^{0}(X, L) \supset H^{0}(X, E) \neq 0$, we know that $c_{1}(L) \geq 0$. Moreover $c_{1}(T)=0$ and $c_{2}(T) \leq 0$ as was shown in the proof of Theorem 2.5. Hence we know that $c_{1}(E)=c_{1}(L) \geq 0$ and $c_{2}(E)$ $=-c_{2}(T) \geq 0$. q.e.d.

For convenience sake let us introduce the notion of cotype.

DEFINITION. Let $X$ be a non-singular projective variety defined over $k$ and let $H$ be a very ample linebundle on $X$. Let $\beta_{1}, \cdots, \beta_{r-1}$ be a sequence of $r-1$ rational numbers. Then a torsion free $\mathcal{O}_{X}$-module $E$ of rank $r$ is called of cotype $\beta_{1}, \cdots, \beta_{r-1}$ (with respect to $H$ ) if and only if for any coherent $\mathcal{O}_{X}$-submodule $G$ of rank $s(1 \leq s \leq r-1)$, the following inequalities are satisfied;

$$
d(E, H) / r(E)+\beta_{s} \geq d(G, H) / r(G) .
$$

By virtue of Lemma $1.2 E$ is of cotype $\beta_{1}, \cdots, \beta_{r-1}$ if and only if it is of type $\beta_{r-1} /(r-1), \cdots, s \beta_{r-s} /(r-s), \cdots,(r-1) \beta_{1}$.

Let us come back to the surface case.

LEMMA 3.2. If a vector bundle $E$ of rank $r$ on $X$ is not of cotype $\beta_{1}, \cdots, \beta_{r-1}$, then there exists a locally free $\mathcal{O}_{X}$-submodule $G$ of rank $s$ for some $1 \leq s \leq r-1$ such that $G$ is of cotype $\beta_{1}-\beta_{s}, \cdots, \beta_{s-1}-\beta_{s}, E / G$

2) This means that for the rational equivalence class $c_{1}(E)$, the complete linear system $\left|c_{1}(E)\right|$ is not empty. 
is torsion free and that $d(G, H) / r(G)>d(E, H) / r(E)+\beta_{s} . \quad$ (If $s=1$, then the condition about the cotype of $G$ is automatically satisfied).

Proof. Since $E$ is not of cotype $\beta_{1}, \cdots, \beta_{r-1}$, there is an $\mathcal{O}_{X}$-submodule $F$ of $E$ of rank $s\left(1 \leq{ }^{\exists} s \leq r-1\right)$ such that $d(F, H) / r(F)>d(E, H) / r(E)$ $+\beta_{s}$. If $E^{\prime}=E / F, T$ is the torsion part of $E^{\prime}$ and if $E^{\prime \prime}=E^{\prime} / T$, then there are two exact sequences;

$$
\begin{aligned}
& 0 \longrightarrow F \longrightarrow E \stackrel{p}{\longrightarrow} E^{\prime} \longrightarrow 0 \\
& 0 \longrightarrow T \longrightarrow E^{\prime} \longrightarrow E^{\prime \prime} \longrightarrow 0
\end{aligned}
$$

Put $F^{\prime}=p^{-1}(T)$. Since $c_{1}\left(F^{\prime}\right)=c_{1}(F)+c_{1}(T)$ and since $c_{1}(T) \geq 0$, we get $d\left(F^{\prime}, H\right) / r\left(F^{\prime}\right) \geq d(F, H) / r(F)>d(E, H) / r(E)+\beta_{s}$. Let $x_{1}, \cdots, x_{n}$ be the pinch points of $F^{\prime}$ and $i: U=X-\left\{x_{1}, \cdots, x_{n}\right\} \rightarrow X$ be the natural inclusion. Then $F^{\prime \prime}=i_{*} i^{*}\left(F^{\prime}\right)$ is a locally free $\mathcal{O}_{X}$-submodule of $E=i_{*} i^{*}(E)$. Look at the following diagram;

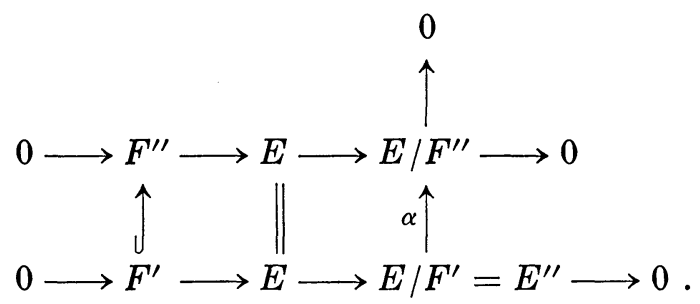

Since $E^{\prime \prime}$ is torsion free and since $\left(E / F^{\prime \prime}\right)\left|U \stackrel{\alpha \underline{\longrightarrow}}{\longrightarrow} E^{\prime \prime}\right| U$, we know that $\operatorname{Supp}(\operatorname{ker}(\alpha)) \subset\left\{x_{1}, \cdots, x_{n}\right\}$, whence $\operatorname{ker}(\alpha)=0$. Thus $\alpha$ is an isomorphism, which means that $F^{\prime}=F^{\prime \prime}$. If $F^{\prime}$ is of cotype $\beta_{1}-\beta_{s}, \cdots, \beta_{s-1}-\beta_{s}$, then it is one of the desired submodules. Assume that $F^{\prime}$ is not of cotype $\beta_{1}-\beta_{s}, \cdots, \beta_{s-1}-\beta_{s}$. Then by induction on $r(E)$ we obtain a locally free $\mathcal{O}_{X}$-submodule $G$ of $F^{\prime}$ of rank $t$ such that $G$ is of cotype $\left(\beta_{1}-\beta_{s}\right)-\left(\beta_{t}-\beta_{s}\right)=\beta_{1}-\beta_{t}, \cdots,\left(\beta_{t-1}-\beta_{s}\right)-\left(\beta_{t}-\beta_{s}\right)=\beta_{t-1}-\beta_{t}, \quad F^{\prime} / G$ is torsion free and that $d(G, H) / r(G)>d\left(F^{\prime}, H\right) / r\left(F^{\prime}\right)+\beta_{t}-\beta_{s}>d(E, H) / r(E)$ $+\beta_{t}$. Since $F^{\prime} / G$ and $E / F^{\prime}$ are torsion free, so is $E / G$. Thus $G$ is one of the desired submodules.

q.e.d.

LEMMA 3.3. Let $R$ be a complete discrete valuation ring over a field $K(K \supset k), L(o r, M)$ be the quotient field (or, the residue field, resp.) of $R$ and let $g$ (or, $s$ ) be the generic point (or, the closed point, resp.) of $\operatorname{Spec}(R)$. If $F$ is a vector bundle of rank $r$ on $Y=X_{K} \times_{K} \operatorname{Spec}(R)$ and if $\bar{F}_{g}=F \otimes_{O Y} \bar{L}$ is not of type $\alpha_{1}, \cdots, \alpha_{r-1}$ on $X_{\bar{L}}$ with respect to $H_{\bar{L}}$, 
then so is $\bar{F}_{s}=F \otimes_{o r} \bar{M}$ on $X_{\bar{M}}$ with respect to $H_{\bar{M}}$, where $\bar{L}($ or, $\bar{M})$ is the algebraic closure of $L$ (or, $M$, resp.).

Proof. By the assumption there is a coherent quotient $\mathcal{O}_{X_{\bar{L}}}$-module $E$ of $\bar{F}_{g}$ of rank $t\left(1 \leq{ }^{\exists} t \leq r-1\right)$ such that $d\left(\bar{F}_{g}, H_{\bar{L}}\right) / r\left(\bar{F}_{q}\right)-\alpha_{t}$ $>d\left(E, H_{\bar{L}}\right) / r(E)$. Then there exist a finite algebraic extension $L^{\prime}$ of $L$ and a coherent quotient $\mathcal{O}_{X_{L^{\prime}}}$-module $E^{\prime}$ of $F_{g^{\prime}}=F \otimes_{O_{Y}} L^{\prime}$ such that $E=E^{\prime} \otimes_{L^{\prime}} \bar{L}$. Take a discrete valuation ring $R^{\prime}$ with $Q\left(R^{\prime}\right)=L^{\prime}$ which extends $R$. Then the residue field of $R^{\prime}$ is a finite algebraic extension $L^{\prime}$ of $L$ and a coherent quotient $\mathcal{O}_{X_{L^{\prime}}}$-module $E^{\prime}$ of $F_{g}^{\prime}=F \otimes_{O_{Y}} L^{\prime}$ such that $E=E^{\prime} \otimes_{L^{\prime}} \bar{L}$. Take a discrete valuation ring $R^{\prime}$ with $Q\left(R^{\prime}\right)=L^{\prime}$ which extends $R$. Then the residue field of $R^{\prime}$ is a finite algebraic extension $M^{\prime}$ of $M$. Put $Y^{\prime}=X_{K} \times{ }_{K} \operatorname{Spec}\left(R^{\prime}\right)$ and $F^{\prime}=F_{Y^{\prime}}$. Then there is a coherent quotient $\mathcal{O}_{Y}{ }^{\prime}$-module $G$ of $F^{\prime}$ such that $G$ is $R^{\prime}$-flat and $G_{g}=E^{\prime}$ ([2], Lemma 3.7). Since $G$ is $R^{\prime}$-flat, $d\left(E, H_{\bar{L}}\right)=d\left(G_{g}, H_{L^{\prime}}\right)$ $=d\left(G_{s}, H_{M^{\prime}}\right)=d\left(G_{s} \otimes_{M^{\prime}} \bar{M}, H_{\bar{M}}\right) \quad$ and $\quad t=r(E)=r\left(G_{s} \otimes_{M^{\prime}} \bar{M}\right)$. Thus $d\left(\bar{F}_{s}, H_{\bar{M}}\right) / r\left(\bar{F}_{s}\right)-\alpha_{t}>d\left(G_{s} \otimes_{M^{\prime}} \bar{M}, H_{\bar{M}}\right) / r\left(G_{s} \otimes_{M^{\prime}} \bar{M}\right)$.

q.e.d.

Now let us prove our main theorem in this section.

THEOREM 3.4. Let $X$ be a non-singular projective surface with a very ample linebundle $H, T$ be a scheme locally of finite type over $k$

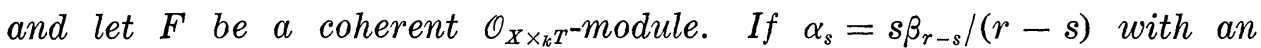
ascending sequence of rational numbers $\beta_{1}, \cdots, \beta_{r-1}$ and if $F$ is T-flat, then the set $S_{\{\beta\}}=\left\{t \in T(k) \mid F_{t}\right.$ is locally free and of type $\alpha_{1}, \cdots, \alpha_{r-1}$ with respect to $H\}$ is that of k-rational points of an open set of $T$.

Proof. Since the problem is a local property with respect to $T$, we may assume that $T$ is of finite type. Since $T^{\prime}=\left\{t \in T \mid F_{t}\right.$ is locally free $\}$ is open, we may assume that $F$ is locally free ([12], p. 320). There is an integer $n$ such that $F_{t}(n)$ is generated by its global sections for any $t \in T$. Hence replacing $F$ by $F \otimes p_{1}{ }^{*}\left(H^{\otimes n}\right)$, we may assume that $F_{t}$ is generated by its global sections for any $t \in T$. We may assume also that $T$ is connected, whence $c_{1}\left(F_{t}\right), c_{2}\left(F_{t}\right), r\left(F_{t}\right)$ are invariable (numerical equivalence). Let us prove our theorem by induction on $r\left(\boldsymbol{F}_{t}\right)$. First of all note that a torsion free $\mathcal{O}_{X}$-module is of type $\alpha_{1}, \cdots, \alpha_{r-1}$ if and only if it is of cotype $\beta_{1}, \cdots, \beta_{r-1}$ (Lemma 1.2). Now if $r\left(F_{t}\right)=1$, then there is nothing to prove. Assume that $r=r\left(F_{t}\right) \geq 2$. If we put $\alpha=d\left(F_{t}, H\right) / r$, then $\alpha$ is independent of $t$ and $\alpha \geq 0$ by virtue of Corol- 
lary 3.1.1. Let $B_{s}$ be the set of locally free $\mathcal{O}_{X}$-modules $E$ of rank $s$ such that 1) $s<r, 2)$ for some $t \in T(k), E$ is an $\mathcal{O}_{X}$-submodule of $F_{t}$ and $F_{t} / E$ is torsion free, 3) $E$ is of cotype $\gamma_{1}^{s}, \cdots, \gamma_{s-1}^{s}\left(\gamma_{i}^{s}=\beta_{i}-\beta_{s}\right)$, 4) $d(E, H) / s>\alpha+\beta_{s}$. Let us show that $B=\bigcup_{s=1}^{r-1} B_{s}$ is bounded. By virtue of Corollary 2.5.1 we have only to prove that $c_{1}(E), c_{2}(E), E \in B$ range over a finite number of numerical equivalence classes. For any $E \in B$ take a point $t \in T(k)$ such that $E \subset F_{t}$ and $F_{t} / E$ is torsion free. Since $F_{t}$ is generated by its global sections, so is $G=F_{t} / E$. Then by virtue of Corollary 3.1.1 we get that $c_{1}(G) \geq 0, c_{2}(G) \geq 0$. On the other hand, since $\min \left(\alpha+\beta_{1},(r-1)\left(\alpha+\beta_{1}\right)\right)<d(E, H)=d\left(F_{t}, H\right)-d\left(c_{1}(G), H\right)$, $d\left(c_{1}(G), H\right)$ is smaller than some constant $a$. Hence the set of numerical equivalence classes of $c_{1}(G)$ 's is finite, whence so is the set $\mathscr{N}=\left\{c_{1}(E)\right.$ $\left.=c_{1}\left(F_{t}\right)-c_{1}(G) \mid E \in B\right\} /$ (numerical equivalence). As for the second Chern classes since $\quad c_{2}(E)=c_{2}\left(F_{t}\right)-c_{2}(G)-\left(c_{1}(E), c_{1}\left(F_{t}\right)-c_{1}(E)\right) \leq c_{2}\left(F_{t}\right)-$ $\left(c_{1}(E), c_{1}\left(F_{t}\right)-c_{1}(E)\right)$ and since $\#(\mathscr{N})<\infty$, there is a constant $a^{\prime}$ such that $c_{2}(E) \leq a^{\prime}$ for any $E$ in $B$. Moreover, since every $E$ in $B_{s}$ is of cotype $\gamma_{1}^{s}, \cdots, \gamma_{s-1}^{s}$ and $\sharp(\mathscr{N})<\infty$, there is a constant $a^{\prime \prime}$ such that for any $E$ in $B, a^{\prime \prime} \leq c_{2}(E)$ by virtue of Corollary 2.5.2. Therefore there are an algebraic $k$-scheme $P$ and a locally free sheaf $F^{\prime}$ on $X \times{ }_{k} P$ such that $B \subset\left\{F_{p}^{\prime} \mid p \in P(k)\right\}$. Since we may assume that $r\left(F_{t}\right)>r\left(F_{p}^{\prime}\right)$ for any $p \in P$, the set $\bigcup_{s=1}^{r-1}\left\{q \in P(k) \mid F^{\prime}{ }_{q}\right.$ is of cotype $\gamma_{1}^{s}, \cdots, \gamma_{s-1}^{s}$ and $d\left(F^{\prime}{ }_{q}, H\right) / r\left(F^{\prime}{ }_{q}\right)$ $\left.>\alpha+\beta_{s}\right\}$ is that of $k$-rational points of an open set $Q$ of $P$ by virtue of the induction assumption. Put $F^{\prime \prime}=F^{\prime} \mid Q$. Let $S^{\prime}$ be the set $\{t \in$ $T(k) \mid$ Hom $_{\mathscr{O}_{X}}\left(F^{\prime \prime}{ }_{q}, F_{t}\right) \neq 0$ for some $\left.q \in Q(k)\right\}$. For a $t \in T(k)$, if $F_{t}$ is not of cotype $\beta_{1}, \cdots, \beta_{r-1}$, then $F_{t} \supset E$ with some $E \in B$ by virtue of Lemma 3.2. This implies that $\operatorname{Hom}_{\mathscr{o}_{X}}\left(F^{\prime \prime}{ }_{q}, F_{t}\right) \neq 0$ for a $q \in Q(k)$ because $B \subset$ $\left\{F^{\prime \prime}{ }_{q} \mid q \in Q(k)\right\}$. Thus we get that $T(k)-S_{\{\beta\}} \subset S^{\prime}$. Conversely, suppose that there is a non-zero homomorphism $\mu: F^{\prime \prime}{ }_{q} \rightarrow F_{t}$ for some $q \in Q(k)$ and $t \in T(k)$. If we put $E=\mu\left(F^{\prime \prime}{ }_{q}\right)$, then $E$ is torsion free with $r(E)$ $<r\left(F_{t}\right)$. Since $F^{\prime \prime}{ }_{q}$ is of cotype $\gamma_{1}^{s}, \cdots, \gamma_{s-1}^{s}$, we have that $d(E, H) / r(E)$ $\geq d\left(F^{\prime \prime}{ }_{q}, H\right) / s-u\left(\beta_{s-u}-\beta_{s}\right) /(s-u)>\alpha+\beta_{s}-u\left(\beta_{s-u}-\beta_{s}\right) /(s-u) \geq \alpha+\beta_{u}$ because of the condition (4) on $B$. We know therefore that $F_{t}$ is not of cotype $\beta_{1}, \cdots, \beta_{r-1}$, whence $S^{\prime} \subset T(k)-S_{\{\beta\}}$. Consequently $S^{\prime}=T(k)$ $-S_{\{\beta\}}$. Now let us consider the locally free sheaf $\tilde{F}=p_{12} *(F) \otimes p_{13} *\left(F^{\prime \prime \vee}\right)$ on $X \times{ }_{k} T \times{ }_{k} Q$. For a $k$-rational point $(t, q)$ of $T \times{ }_{k} Q, \tilde{F}_{(t, q)} \cong F_{t} \otimes_{\mathcal{O}_{X}} F^{\prime \prime}{ }_{q}$ $\cong \mathscr{H}_{o m_{0 X}}\left(F^{\prime \prime}{ }_{q}, F_{t}\right)$. Hence $H^{0}\left(X, \tilde{F}_{(t, q)}\right) \cong \operatorname{Hom}_{0_{X}}\left(F^{\prime \prime}{ }_{q}, F_{t}\right)$. By virtue of upper semi-continuity of $h^{0}\left(\tilde{F}_{(t, q)}\right), \Gamma=\left\{x \in T \times{ }_{k} Q \mid h^{0}\left(F_{x}\right) \neq 0\right\}$ is closed 
in $T \times{ }_{k} Q$. Then $\pi(T)$ is a constructible set of $T$, where $\pi$ is the natural projection of $T \times{ }_{k} Q$ to $T$. By virtue of the above argument we know that $\pi(\Gamma)(k)=T(k)-S_{\{\beta\}}$. On the other hand, $\pi(T)$ is closed under specializations by virtue of Lemma 3.3. Therefore $\pi(\Gamma)$ is closed in $T$, whence $U=T-\pi(\Gamma)$ is open in $T$. Moreover $U(k)=S_{\{\beta\}}$. q.e.d.

Corollary 3.4.1. Let $F$ and $T$ be the same as in Theorem 3.4. Put $S_{0}=\left\{t \in T(k) \mid F_{t}\right.$ is locally free and semi-stable $\}$ and $S=\left\{t \in T(k) \mid F_{t}\right.$ is locally free and stable\}. Then $S_{0}$ and $S$ are sets of k-rational points of open sets of $T$.

\section{§ 4. Moduli of stable vector bundles of rank 2 on an algebraic surface.}

Our aim of this section is to construct coarse moduli schemes of stable vector bundles of rank 2 on a non-singular projective surface. We shall maintain the notation in the preceding two sections $\left(X, H, h, K_{X}\right.$ etc.).

LeMma 4.1. Let $\mathscr{F}$ be a family of vector bundles of rank 2 on $X$ with fixed Chern classes $c_{1}, c_{2}$. Assume that for any $E \in \mathscr{F}$, (i) $d(E, H)$ - $d\left(K_{X}, H\right)>0$, (ii) $h^{1}(E(n))=h^{2}(E(n))=0$ for any non-negative integer $n$. Then there exists an integer $m_{0}$ such that $h^{0}(L(m))<h^{0}(E(m)) / 2$ for any integer $m \geq m_{0}, E \in \mathscr{F}$ and for any invertible $\mathcal{O}_{X}$-submodule $L$ of $E$ with $d(L, H)<d(E, H) / 2$.

Proof. 1) Assume that $d(L, H)<0$. Let us consider the following exact sequence;

$$
0 \longrightarrow L \longrightarrow L(m) \longrightarrow L(m) \otimes_{O_{X}} \mathcal{O}_{C} \longrightarrow 0,
$$

where $C$ is a non-singular member of $\left|H^{\otimes m}\right|$. Since the genus of $C$ is $\left(m^{2} h+m d\left(K_{X}, H\right)\right) / 2+1$, we get $h^{0}\left(L(m) \otimes_{O_{X}} \mathcal{O}_{C}\right) \leq A(m)=\max \left\{m^{2} h / 2\right.$ $\left.+m\left(d(L, H)-d\left(K_{X}, H\right) / 2\right),\left(m^{2} h+m d(L, H)\right) / 2,0\right\}$ by Riemann-Roch theorem and Cliford's theorem. Moreover $h^{0}(L(m)) \leq h^{0}\left(L(m) \otimes_{O_{X}} \mathcal{O}_{C}\right)$ because the assumption $d(L, H)<0$ implies that $h^{0}(L)=0$. Thus we have that $h^{0}(L(m)) \leq A(m)$. On the other hand, our assumption (ii) and RiemannRoch theorem imply

$$
\begin{aligned}
h^{0}(E(m)) / 2= & m^{2} h / 2+m\left(d\left(c_{1}, H\right)-d\left(K_{X}, H\right)\right) / 2 \\
& +\left(c_{1}-K_{X}, c_{1}\right) / 4-c_{2} / 2+\chi\left(\mathcal{O}_{X}\right) \\
= & B(m) .
\end{aligned}
$$


Thus we obtain

$$
\begin{aligned}
& h^{0}(E(m)) / 2-h^{0}(L(m)) \geq \min \left[m\left\{d\left(c_{1}, H\right) / 2-d(L, H)\right\}\right. \\
& \quad+\left(c_{1}-K_{X}, c_{1}\right) / 4-c_{2} / 2+\chi\left(\mathcal{O}_{X}\right), m\left\{d\left(c_{1}, H\right)\right. \\
& \left.\quad-d\left(K_{X}, H\right)-d(L, H)\right\} / 2+\left(c_{1}-K_{X}, c_{1}\right) / 4 \\
& \left.\quad-c_{2} / 2+\chi\left(\mathcal{O}_{X}\right), B(m)\right] .
\end{aligned}
$$

Since $d(L, H)<0, d\left(c_{1}, H\right)-d\left(K_{X}, H\right)>0$ and since $d\left(c_{1}, H\right) / 2-d(L, H)$ $>0$, there exists an integer $m_{1}$ (independent of $L$ and $E$ ) such that the right hand side of the above inequality is positive for any $m \geq m_{1}$, whence $h^{0}(L(m))<h^{0}(E(m)) / 2$ for any $m \geq m_{1}$.

2) Assume that $d(L, H) \geq 0$. Let us consider the following exact sequence ;

$$
0 \longrightarrow L(m-1) \longrightarrow L(m) \longrightarrow L(m) \otimes_{\mathcal{O}_{X}} \mathcal{O}_{D} \longrightarrow 0,
$$

where $D$ is a non-singular member of $|H|$. Since the genus $g$ of $D$ is $\left(h+d\left(K_{X}, H\right)\right) / 2+1$, there exists a positive integer $m_{2}$ such that $\operatorname{deg}\left(L(m) \otimes_{\mathcal{O}_{X}} \mathcal{O}_{D}\right) \geq 2 g-1$ for any $L$ with $d(L, H) \geq 0$ and for any $m>m_{2}$. On the other hand, since $d\left(c_{1}, H\right) / 2>d(L, H)$, there exists an integer $m_{3}$ such that $d(L(m), H)<0$ for any $m \leq m_{3}$ and $L$, whence $h^{0}(L(m))=0$ for any $m \leq m_{3}$ and $L$. Moreover the assumption that $d(L, H)<d\left(c_{1}, H\right) / 2$ implies that there is a constant $c$ such that $h^{0}(L(m)$ $\left.\otimes_{\mathcal{O}_{X}} \mathcal{O}_{D}\right) \leq c$ for any $m_{3}<m \leq m_{2}$ and $L$. If $m>m_{2}$, then $h^{0}\left(L(m) \otimes_{\mathcal{O}_{X}} \mathcal{O}_{D}\right)$ $=m h+d(L, H)-\left(h+d\left(K_{X}, H\right)\right) / 2$ because $L(m) \otimes_{\mathcal{O}_{X}} \mathcal{O}_{D}$ is not special. On the other hand, we have that $h^{0}(L(m)) \leq h^{0}(L(m-1))+h^{0}\left(L(m) \otimes_{\mathcal{O}_{X}} \mathcal{O}_{D}\right)$. Thus for any $m>m_{2}$,

$$
\begin{aligned}
h^{0}(L(m)) \leq & \sum_{i=m_{3}+1}^{m} h^{0}\left(L(i) \otimes_{o_{X}} \mathcal{O}_{D}\right) \\
\leq & \left(m_{2}-m_{3}\right) c-m_{2}{ }^{2} h / 2-m_{2} d(L, H)+m_{2} d\left(K_{X}, H\right) / 2 \\
& +m^{2} h / 2+m\left\{d(L, H)-d\left(K_{X}, H\right) / 2\right\} \\
\leq & \left(m_{2}-m_{3}\right) c+m_{2} d\left(K_{X}, H\right) / 2+m^{2} h / 2 \\
& +m\left\{d(L, H)-d\left(K_{X}, H\right) / 2\right\} \\
= & m^{2} h / 2+m\left\{d(L, H)-d\left(K_{X}, H\right) / 2\right\}+c^{\prime},
\end{aligned}
$$

where $c^{\prime}=\left(m_{2}-m_{3}\right) c+m_{2} d\left(K_{X}, H\right) / 2$. We get therefore

$$
\begin{aligned}
h^{0}(E(m)) / 2-h^{0}(L(m)) \geq & m\left\{d\left(c_{1}, H\right) / 2-d(L, H)\right\} \\
& +\left(c_{1}-K_{X}, c_{1}\right) / 4-c_{2} / 2+\chi\left(\mathcal{O}_{X}\right)-c^{\prime} .
\end{aligned}
$$

Since $d\left(c_{1}, H\right) / 2-d(L, H)>0$, we obtain an integer $m_{4}>m_{2}$ such that 
the right hand side of the above inequality is positive for any $m \geq m_{4}$ and $L$.

Now if one puts $m_{0}=\max \left(m_{1}, m_{4}\right)$, then it satisfies the condition in the lemma.

q.e.d.

LEMMA 4.2. Let $E$ be a vector bundle of rank $r$ on a non-singular projective variety $Y$. Assume that $E$ is generated by its global sections. Let $s_{1}, \cdots, s_{t} \in H^{0}(Y, E)$ be independent at the generic point of $Y$. Then the set $Z=\left\{y \in Y \mid\left(s_{1} \wedge \cdots \wedge s_{t}\right)(y)=0\right\}$ is contained in Supp $(D)$ for some $D \in\left|c_{1}(E)\right|$.

Proof. Since $E$ is generated by its global sections, there are elements $s_{t+1}, \cdots, s_{r}$ of $H^{0}(Y, E)$ such that $s_{1}, \cdots, s_{r}$ form a basis of $E$ at the generic point of $Y$. Obviously $Z \subset\left\{y \in Y \mid\left(s_{1} \wedge \cdots \wedge s_{r}\right)(y)=0\right\}$ and hence we may assume that $t=r$. Then $s_{1} \wedge \cdots \wedge s_{r}$ is a global section of the linebundle $\wedge^{r} E$. On the other hand, $s_{1} \wedge \cdots \wedge s_{r} \neq 0$ because $s_{1}, \cdots, s_{r}$ are independent at the generic point of $Y$. Thus $s_{1} \wedge \cdots \wedge s_{r}$ defines a divisor $D$ in $\left|c_{1}\left(\bigwedge^{r} E\right)\right|$, which completes the proof because $c_{1}\left(\bigwedge^{r} E\right)=c_{1}(E)$ and $\left\{y \in Y \mid\left(s_{1} \wedge \cdots \wedge s_{r}\right)(y)=0\right\}=\operatorname{Supp}(D) . \quad$ q.e.d.

Let $B\left(c_{1}, c_{2}\right)$ be the set of isomorphism classes of stable vector bundles of rank 2 on a non-singular projective surface $X$ with Chern classes $c_{1}, c_{2}$ (modulo numerical equivalence class). As was shown (Corollary 2.5.1), $B\left(c_{1}, c_{2}\right)$ is a bounded family. Hence there is an integer $n_{0}$ such that $B\left(c_{1}, c_{2}\right)(n)=\left\{E(n) \mid E \in B\left(c_{1}, c_{2}\right)\right\}$ satisfies the conditions (i), (ii) in Lemma 4.1 for any $n \geq n_{0}$. Then by virtue of Lemma 4.1 we get an integer $m_{0}$ such that for any $m \geq m_{0}, B\left(c_{1}, c_{2}\right)(m)$ satisfies the following conditions (a), (b) ;

a) For any $E \in B\left(c_{1}, c_{2}\right)(m), E$ is generated by its global sections and $h^{1}(E)=h^{2}(E)=0$.

b) For any $E \in B\left(c_{1}, c_{2}\right)(m)$ and any invertible $\mathcal{O}_{X}$-submodule $L$ of $E, h^{0}(L)<h^{0}(E) / 2$.

Let us fix an integer $m \geq m_{0}$. Since $h^{0}(E)=p$ is constant for any $E \in B\left(c_{1}, c_{2}\right)(m)$ and since $E$ is generated by its global sections, every vector bundle in $B\left(c_{1}, c_{2}\right)(m)$ is a quotient of $\mathcal{O}_{X}^{\otimes p}$. Meanwhile if $\tilde{F}$ is the

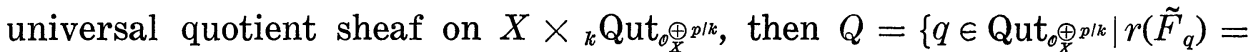
$\left.2, c_{1}\left(\tilde{F}_{q}(-m)\right)=c_{1}, c_{2}\left(\tilde{F}_{q}(-m)\right)=c_{2}\right\}$ is a union of a finite number of connected components of Qut $_{\sigma_{X}^{\oplus p / k}}$ and hence $Q$ is projective over $k$. Obviously $B\left(c_{1}, c_{2}\right)(m)$ is contained in the set of $k$-rational points of $Q$ (that 
is, for any $E \in B\left(c_{1}, c_{2}\right)(m)$, there is a $k$-rational point $q$ of $Q$ such that the isomorphism class of $\tilde{F}_{q}$ is $\left.E\right)$. Moreover $\operatorname{Aut}_{X}\left(\mathcal{O}_{\bar{X}}^{\oplus p}\right)=G L(p, k)$ naturally acts on $Q$ and the center of $G L(p, k)$ stabilizes every point of $Q$. Thus $G=P G L(p, k)=G L(p, k) / G_{m}$ acts on $Q$. Now $R_{0}=\left\{q \in Q \mid \tilde{F}_{q}\right.$ is locally free $\}$ is a $G$-stable open subset of $Q$ and $F=\left.\tilde{F}\right|_{X \times_{k} R_{0}}$ is a locally free $\mathcal{O}_{X \times{ }_{k} R_{0}}$-module of rank 2 ([12]) Proposition 6.1). Let $\mathfrak{n}$ be an ordered set of $N$ distinct points $x_{1}, \cdots, x_{N}$ on $X$. Then as in [12] we obtain a morphism $\tau(\mathfrak{n})$ of $R_{0}$ to $H_{p, 2}^{N}$, where $H_{p, 2}^{N}$ is the product of $N$ copies of the Grassmann variety $H_{p, 2}^{N}$ of 2-dimensional linear quotient spaces of a $p$-dimensional linear space over $k$ (i-th coordinate of $\tau(\mathfrak{n})(q)$ is the quotient vector space of $H^{0}\left(X, \mathcal{O}_{X}^{\oplus p}\right)$ represented by the fibre of $F_{q}$ at $x_{i}$, then $\tau(\mathfrak{n})$ is a morphism because of the universality of Grassmann variety). Clearly $\tau(\mathfrak{n})$ is a $G$-morphism with respect to the natural action of $G$ on $H_{p, 2}^{N}$. If $N$ is sufficiently large and if $x_{1}, \cdots, x_{N}$ are in sufciently general position, then $\tau(\mathfrak{n})$ is injective ([12] $\mathrm{p} 326$ ). If $R$ is the subset of $R_{0}$ consisting of the points $q \in R_{0}$ such that the canonical map $H^{0}\left(X \times{ }_{k} k(b), \mathcal{O}_{X \times k} \underset{k(q)}{\oplus p}\right) \rightarrow H^{0}\left(X \times{ }_{k} k(q), F_{q}\right)$ is bijective, then it is $G$-stable open subset of $R_{0}$. Moreover for $q_{1}, q_{2} \in R, F_{q_{1}}$ and $F_{q_{2}}$ are isomorphic to each other if and only if $q_{1}$ can be transformed to $q_{2}$ by the action of $G$ ([12] Proposition 6.2). The set $\left\{q \in R(k) \mid F_{q}\right.$ is a stable vector bundle $\}$ is that of $k$-rational points of a $G$-stable open subset $R_{s}$ of $R$ by virtue of Corollary 3.4.1. Then the above argument implies that the set of isomorphism classes of $F_{q}, q \in R_{s}(k)$ is just $B\left(c_{1}, c_{2}\right)(m)$.

Now the proof of the following proposition is essentially same as that in [12].

Proposition 4.3. If $N$ is sufficiently large and if $x_{1}, \cdots, x_{N}$ are in sufficiently general position, then $\tau(\mathfrak{n})\left(R_{s}\right)$ is contained in the set of properly stable points (see [8] and [12]) of $H_{p, 2}^{N}$ with respect to the canonical action of $G=P G L(p, k)$ on $H_{p, 2}^{N}$ and the linebundle which defines the Plücker coordinates.

Proof. Take a $k$-rational point $q$ of $R_{s}$ and put $E=F_{q}$. Let $F$ be a proper linear subspace of $V=H^{0}\left(X, \mathcal{O}_{X}^{\oplus p}\right)$ and let $F_{i}$ be the linear subspace of $E\left(x_{i}\right)=E \otimes_{O_{X}} k\left(x_{i}\right)$ generated by $\left\{s\left(x_{i}\right) \mid s \in F\right\}$. We set

$$
\nu(F)=\frac{\left(\sum_{i=1}^{N} r\left(F_{i}\right)\right) / N}{r(F)}-\frac{2}{p},
$$


where $r(F)$ (or, $r\left(F_{i}\right)$ ) is the rank of $F$ (or, $F_{i}$, resp.) over $k$. What we have to prove is that $\nu(F)>0$ for any proper subspace $F$ of $V$ (see [13] p. 328 or [8] Proposition 4.3). Let $L_{1}$ be the $\mathcal{O}_{X}$-submodule of $E$ generated by $F$. Then there is a unique locally free $\mathcal{O}_{X}$-submodule $L(F)$ of $E$ such that $L(F)$ is isomorphic to $L_{1}$ on an open set $U$ of $X$ with codim $(X-U, X) \geq 2$ by virtue of Lemma 2.2. Let us set

$$
\nu(L(F))=\frac{r(L(F))}{r(F)}-\frac{2}{p} .
$$

Then $\nu\left(L\left(F^{\prime}\right)\right) \geq 1 / p^{2}$ because $r\left(F^{\prime}\right) \leq h^{0}\left(L\left(F^{\prime}\right)\right)<p / 2$ or $p$ according to $r(L(F))=1$ or 2 by virtue of the condition (b) on $B\left(c_{1}, c_{2}\right)(m)$. On the other hand, there is a positive constant $\lambda$ such that for every positive integer $N$, there exist $\lambda$-independent 0 -cycles on $X$ of degree $N$ (see [9] Lecture 20, Proposition 1 and note that if $x_{1}, \cdots, x_{N}$ are in sufficiently general position, then $\sum_{i=1}^{N} x_{i}$ is $\lambda$-independent). Take a $\lambda$-independent cycle $\sum_{i=1}^{N} x_{i}$ of degree $N$ and put $\mathfrak{n}=\left(x_{1}, \cdots, x_{N}\right)$. Since $r\left(F_{i}\right)<r(L(F))$ if and only if $\left\{s\left(x_{i}\right) \mid s \in F\right\}$ generates a vector subspace of $E\left(x_{i}\right)$ with rank less than $r(L(F))$, we know that $r\left(F_{i}\right)<r(L(F))$ only if

1) in the case of $r(L(F))=1, s\left(x_{i}\right)=0$ for a fixed non-zero element $s$ of $F$,

$2)$ in the case of $r(L(F))=2,\left(s_{1} \wedge s_{2}\right)\left(x_{i}\right)=0$ for fixed general two elements $s_{1}, s_{2}$ of $F$ (satisfying the condition of Lemma 4.2).

In both cases, $r\left(F_{i}\right)<r(L(F))$ only if $x_{i}$ is contained in Supp $(D)$ for some fixed $D \in\left|c_{1}(E)\right|$ by virtue of Lemma 4.2. Thus we know that [number of $x_{i}$ 's with $r\left(F_{i}\right)<r(L(F))$ ] $\leq$ [number of $x_{i}$ 's contained in Supp $(D)$ ] $\leq \lambda d(D, H)^{2}=\lambda d\left(c_{1}, H\right)^{2}$. We get therefore

$$
0 \leq \nu(L(F))-\nu(F)=\frac{\left(\sum_{i=1}^{N}\left(r(L(F))-r\left(F_{i}\right)\right)\right) / N}{r(F)} \leq \frac{2 \lambda d\left(c_{1}, H\right)^{2}}{N r(F)} .
$$

Thus if $N>2 p^{2} \lambda d\left(c_{1}, H\right)^{2}$, then $0 \leq \nu(L(F))-\nu(F)<1 / p^{2}$ for any $F$. Hence for any proper linear subspace $F$ of $V$, we get that $\nu(F)>0$ because $\nu(L(F)) \geq 1 / p^{2}$.

Fix a $k$-rational point $x$ of $X$ and let $i_{x}$ be the closed immersion $Q \rightarrow X \times{ }_{k} Q$ defined by $x$. Put $G=i_{x}{ }^{*}(\tilde{F})$, then we get an exact sequence;

$$
0 \longrightarrow K \longrightarrow \mathcal{O}_{Q}^{\oplus p} \longrightarrow G \longrightarrow 0 \text {. }
$$

Let $V$ be a 2-dimensional quotient vector space of $H^{0}\left(X, \mathcal{O}_{X}^{\oplus p}\right)$ and let 
$v=\left(v_{i_{1}, \cdots, i_{p-2}}\right)$ be the Plücker coordinates of $V$ with respect to a system of coordinates $e_{1}, \cdots, e_{p}$ of $H^{0}\left(X, \mathcal{O}_{X}^{\oplus p}\right)$ which can be regarded as a free basis of $\mathcal{O}_{X}^{\oplus p}$. For a point $q$ of $Q$ and an affine open neighborhood $U$ of $q$ in $Q$, take a set of generator $\left\{k_{i}=\sum k_{i j} e_{j}\right\}$ of $\Gamma(U, K)$, then $V$ is a quotient space of the quotient vector space of $H^{0}\left(X, \mathcal{O}_{X}^{\oplus p}\right)$ represented by the fibre of $\tilde{F}_{t}, t \in U$ at $x$ if and only if $k_{i} \wedge \sum v_{i_{1}, \cdots, i_{p-2}} e_{i_{1}} \wedge \cdots \wedge e_{i_{p-2}}$ $\equiv 0 \bmod \mathrm{m}_{t}$, that is, $\sum_{\ell=1}^{p-1} k_{i_{j_{\ell}}}(t) v_{j_{1}, \ldots, \grave{j}_{\ell}, \cdots, j_{p-1}}=0$ for any $i$ and any $(p-1)$ ple $\left(j_{1}, \cdots, j_{p-1}\right)$ with $1 \leq j_{1}<\cdots<j_{p-1} \leq p$. Thus the set $\Gamma_{x}=\{(q, V)$ $\in Q \times{ }_{k} H_{p, 2} \mid V$ is a quotient space of the quotient vector space of $H^{0}\left(X, \mathcal{O}_{X}^{\oplus p}\right)$ represented by the fibre of $\tilde{F}_{q}$ at $\left.x\right\}$ is a closed subset of $Q \times{ }_{k} H_{p, 2}$. Similarly for $\mathfrak{n}=\left(x_{1}, \cdots, x_{N}\right), \Gamma_{\mathfrak{n}}=\left\{\left(q, V_{1}, \cdots, V_{N}\right) \in Q \times{ }_{k} H_{p, 2}^{N}\right.$ $\mid V_{i}$ is a quotient space of the quotient vector space of $H^{0}\left(X, \mathcal{O}_{X}^{\oplus p}\right)$ represented by the fibre of $\tilde{F}_{q}$ at $x_{i}$ for every $i$ \} is also a closed subset of $Q \times{ }_{k} H_{p, 2}^{N}$.

LEMMA 4.4. Let $\Phi_{\mathfrak{n}}$ be the correspondence of $Q$ to $H_{p, 2}^{N}$ defined by the above $\Gamma_{\mathfrak{n}}$ witn $\mathfrak{n}=\left(x_{1}, \cdots, x_{N}\right)$. If $N$ is sufficiently large and if $x_{1}, \cdots, x_{N}$ are in sufficiently general position, then for any $q \in Q-R_{0}$, $\Phi_{\mathrm{n}}(q) \cap \tau(\mathfrak{n})\left(R_{0}\right)=\phi$.

Proof. Take some $N$ and $x_{1}, \cdots, x_{N}$. Let $p_{1}$ (or, $p_{2}$ ) be the projection of $\Gamma_{\mathfrak{n}}\left(\mathfrak{n}=\left(x_{1}, \cdots, x_{N}\right)\right)$ to $Q$ (or, $H_{p, 2}^{N}$, resp.). For the diagonal scheme $\Delta$ of $H_{p, 2}^{N} \times{ }_{k} H_{p, 2}^{N}$, we set $B_{n}=\left[\left(p_{1} \times p_{1}\right)\left\{\left(p_{2} \times p_{2}\right)^{-1}(\Delta)\right\}\right] \cap\left\{R_{0} \times(Q\right.$ $\left.\left.-R_{0}\right)\right\}$. Then since $H_{p, 2}^{N}$ is projective over $k,\left(p_{1} \times p_{1}\right)\left\{\left(p_{2} \times p_{2}\right)^{-1}(\Delta)\right\}$ is closed in $Q \times{ }_{k} Q$ and hence $B_{\mathfrak{n}}$ is closed in $R_{0} \times{ }_{k}\left(Q-R_{0}\right)$. It is sufficient to prove that $B_{\mathfrak{n}}=\phi$ if $N$ is sufficiently large and if $x_{1}, \cdots, x_{N}$ are in sufficiently general position. We claim

Sublemma 4.5. Let $S$ be a non-singular projective variety of dimension $n$ and let $E_{1}, E_{2}$ be two distinct quotient coherent $\mathcal{O}_{S}$-modules of a locally free $\mathcal{O}_{S}$-module $E_{0}$ with the same Chern classes $c_{1}, \cdots, c_{n}$ (numerical equivalence) and the same rank $r$. If $E_{1}$ is locally free, then there exists a non-empty open set $U$ of $S$ such that for any point $s$ of $U, E_{1}(s)=E_{1} \otimes_{O_{S}} k(s)$ and $E_{2}(s)=E_{2} \otimes_{o_{S}} k(s)$ are different to each other as quotient vector spaces of $E_{0}(s)=E_{0} \otimes_{\sigma_{S}} k(s)$ and that $E_{2}$ is locally free on $U$.

Proof. Since $S$ is reduced, it is clear that there exists an open set $U_{1}$ on which $E_{2}$ is locally free. Let us consider $Y=P\left(E_{0}\right), W_{1}=\mathbf{P}\left(E_{1}\right)$ and $W_{2}=\mathbf{P}\left(E_{2}\right)$, where $W_{i}$ can be regarded as closed subschemes of $Y$. 
If $F$ is the scheme theoretic intersection of $\left.W_{1}\right|_{U_{1}}$ and $\left.W_{2}\right|_{U_{1}}$ in $\left.Y\right|_{U_{1}}$, then $Z=\left\{s \in U_{1} \mid E_{1}(s)=E_{2}(s)\right.$ as quotient vector spaces of $\left.E_{0}(s)\right\}$ is just $\left\{s \in U_{1} \mid \operatorname{dim} F_{s}=r-1\right\}$. Thus $Z$ is closed in $U_{1}$ by virtue of a theorem of Chevalley (E. G. A. Ch. IV, 13.1.3). Hence we have only to prove that $Z \neq U_{1}$. Assume that $Z=U_{1}$, then $\left.W_{1}\right|_{U_{1}}$ is contained in $\left.W_{2}\right|_{U_{1}}$ as sets. On the other hand, $W_{1}$ is reduced and irreducible because $E_{1}$ is locally free. Thus $W_{1}$ is contained in $W_{2}$ as schemes. Therefore if $\mathcal{O}_{Y}(1)$ is the tautological linebundle of $E_{0}$ and if $\pi$ is the natural projection of of $Y$ to $X$, then we get homomorphisms $\alpha_{1}: \pi_{*}\left(\mathscr{O}_{Y}(1)\right)=E_{0} \rightarrow \pi_{*}\left(\mathcal{O}_{Y}(1)\right.$ $\left.\bigotimes_{O_{Y}} \mathcal{O}_{W_{1}}\right)=E_{1}, \alpha_{2}: E_{0} \rightarrow \pi_{*}\left(\mathcal{O}_{Y}(1) \bigotimes_{O_{Y}} \mathcal{O}_{W_{1}}\right)=E_{2}{ }^{\prime}$ and $\beta: E_{2}{ }^{\prime} \rightarrow E_{1}$ with $\beta \alpha_{2}=\alpha_{1}$. On the other hand, there is a natural homomorphism $\gamma: E_{2} \rightarrow E_{2}^{\prime}$ (E. G. A. Ch. II, 3.3). If $\delta_{i}: E_{0} \rightarrow E_{i}(i=1,2)$ are given homomorphisms, then clearly $\delta_{1}=\alpha_{1}$ and $\gamma \delta_{2}=\alpha_{2}$. Since $\beta \gamma \delta_{2}=\beta \alpha_{2}=\alpha_{1}=\delta_{1}$ is surjective, we know

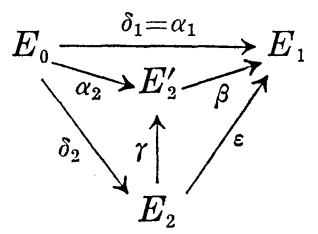

that $\varepsilon=\beta \gamma$ is surjective. If $T$ is the torsion part of $E_{2}$, then $T=\operatorname{ker}(\varepsilon)$ because $E_{1}$ is torsion free, $r\left(E_{1}\right)=r\left(E_{2}\right)$ and $\varepsilon$ is surjective. Thus we get the following exact sequence;

$$
0 \longrightarrow T \longrightarrow E_{2} \stackrel{\varepsilon}{\longrightarrow} E_{1} \longrightarrow 0 \text {. }
$$

This sequence provides the equality $\chi\left(E_{2}(a)\right)=\chi\left(E_{1}(a)\right)+\chi(T(a))$ for every integer $a$. On the other hand, our assumption that $c_{i}\left(E_{1}\right)=c_{i}\left(E_{2}\right)=c_{i}$ (numerical equivalence) implies that for every $a, \chi\left(E_{1}(a)\right)=\chi\left(E_{2}(a)\right)$. We have therefore that for every $a, \chi(T(a))=0$. Take an integer $b$ such that $h^{i}(T(b))=0 \quad(1 \leq i \leq n)$ and that $T(b)$ is generated by its global sections. Then $\chi(T(b))=0$ implies that $H^{0}(S, T(b))=0$ and hence $T(b)$ $=0$. Therefore $\varepsilon$ is an isomorphism. Moreover since $\varepsilon \delta_{2}=\delta_{1}, E_{1}$ is isomorphic to $E_{2}$ as quotient $\mathcal{O}_{S}$-modules of $E_{0}$, which is a contradiction. Thus we get $Z \neq U_{1}$. Then $U=U_{1}-Z$ is one of the desired open sets. q.e.d.

Let us come back to the proof of Lemma 4.4. Assume that $B_{\mathfrak{n}} \neq \phi$ and let $B_{\mathfrak{n}}{ }^{(1)}, \ldots, B_{\mathfrak{n}}{ }^{(t)}$ be irreducible components of $B_{n}$. Take a $k$-rational 
point $\left(q_{i}, q_{i}^{\prime}\right)$ in each $B_{\mathfrak{n}}{ }^{(i)}$ and take an open set $U_{i}$ of $X$ satisfying the conditions in Sublemma 4.5 for $S=X, E_{0}=\mathcal{O}_{\bar{X}}^{\oplus p}, E_{1}=\tilde{F}_{q_{i}}$ and $E_{2}=\tilde{F}_{q_{i^{\prime}}}$ (note that $\tilde{F}_{q_{i}} \not \equiv \tilde{F}_{q_{i^{\prime}}}$ because $\tilde{F}_{q_{i}}$ is locally free but $\tilde{F}_{q_{i^{\prime}}}$ is not so and hence there exists such a $U_{i}$ ). If $y$ is a $k$-rational point of $U=\bigcap_{i=1}^{t} U_{i}$, then $\tilde{F}_{q_{i}}(y) \neq \tilde{F}_{q_{i^{\prime}}}(y)$ as quotient vector spaces of $H^{0}\left(X, \mathcal{O}_{X}^{\oplus p}\right)$ for every $i$. Now put $\mathfrak{n}^{\prime}=\left(x_{1}, \cdots, x_{N}, y\right)$. Then the above result implies that $B_{\mathfrak{n}^{\prime}}$ contains none of $\left(q_{1}, q_{1}^{\prime}\right), \cdots,\left(q_{t}, q_{t}^{\prime}\right)$, whence it does none of $B_{\mathfrak{n}}{ }^{(1)}, \cdots, B_{\mathfrak{n}}{ }^{(t)}$. Thus $\operatorname{dim} B_{\mathfrak{n}^{\prime}}<\operatorname{dim} B_{\mathfrak{n}}$. Applying the above argument repeatedly we get $y_{1}, \cdots, y_{N^{\prime}}$ such that for $\mathfrak{n}_{1}=\left(x_{1}, \cdots, x_{N}, y_{1}, \cdots, y_{N^{\prime}}\right), \operatorname{dim} B_{\mathfrak{n}_{1}}=-1$, that is, $B_{\mathfrak{n}_{1}}=\phi$.

q.e.d.

Our present aim is to prove that $\tau(\mathfrak{n})$ is an immersion and there is a $G$-stable open set $U_{s}$ of $H_{p, 2}^{N}$ such that $\tau(\mathfrak{n})\left(R_{s}\right)$ is closed in $U_{s}$ for $\mathfrak{n}=\left(x_{1}, \cdots, x_{N}\right)$ with a sufficiently large $N$ and $x_{1}, \cdots, x_{N}$ in sufficiently general position.

LEMMA 4.6. If $N$ is sufficiently large and if $x_{1}, \cdots, x_{N}$ are in sufficiently general position, then there exist G-stable open subschemes $U_{s}, U$ and $U_{0}$ of $H_{p, 2}^{N}$ such that for $\mathfrak{n}=\left(x_{1}, \cdots, x_{N}\right)$,

i) $\tau(\mathfrak{n})\left(R_{s}\right) \subset U_{s}, \tau(\mathfrak{H})(R) \subset U$ and $\tau(\mathfrak{n})\left(R_{0}\right) \subset U_{0}$,

ii) the morphisms $\left.\tau(\mathfrak{n})\right|_{R_{s}}: R_{s} \rightarrow U_{s},\left.\tau(\mathfrak{n})\right|_{R}: R \rightarrow U$ and $\tau(\mathfrak{n}): R_{0} \rightarrow U_{0}$ are proper.

Proof. Take $\mathfrak{n}$ such that $\tau(\mathfrak{n})$ is an injective $G$-morphism and the conclusion of Lemma 4.4 holds. Let $f: R_{0} \rightarrow R_{0} \times_{k} H_{p, 2}^{N}$ be the graph morphism of $\tau(\mathfrak{n})$. Then there exists a closed subscheme $\Gamma$ of $Q \times{ }_{k} H_{p, 2}^{N}$ such that $f$ induces an open immersion $f^{\prime}: R_{0} \rightarrow \Gamma$ and that the base space $|\Gamma|$ of $\Gamma$ is contained in $\Gamma_{\mathfrak{n}}$. Since the projection $p_{2}: \Gamma \rightarrow H_{p, 2}^{N}$ is proper, $U_{0}=H_{p, 2}^{N}-p_{2}\left(\Gamma \cap\left(\left(Q-R_{0}\right) \times_{k} H_{p, 2}^{N}\right)\right)$ is an open subscheme of $H_{p, 2}^{N}$. Moreover $U_{0}$ is $G$-stable because $G$ acts naturally on $\Gamma, \Gamma \cap((Q$ $\left.-R_{0}\right) \times_{k} H_{p, 2}^{N}$ ) is a $G$-stable closed subset of $\Gamma$ and because $p_{2}$ is a $G$ morphism. Lemma 4.4 implies that $f^{\prime}\left(R_{0}\right) \subset \Gamma_{U_{0}}=\Gamma \times_{H_{p, 2}^{N}} U_{0}$, whence $f^{\prime}\left(R_{0}\right)=\Gamma_{U_{0}}$. Thus $\tau(\mathfrak{n})=p_{2, U_{0}} \cdot f^{\prime}: R_{0} \rightarrow U_{0}$ is proper because $p_{2, U_{0}}$ is proper and $f^{\prime}: R_{0} \rightarrow \Gamma_{U_{0}}$ is an isomorphism. Put $U_{s}=H_{p, 2}^{N}-p_{2}(\Gamma \cap((Q$ $\left.\left.\left.-R_{s}\right) \times{ }_{k} H_{p, 2}^{N}\right)\right), \quad U=H_{p, 2}^{N}-p_{2}\left(\Gamma \cap\left((Q-R) \times{ }_{k} H_{p, 2}^{N}\right)\right)$, then by a similar argument as above we know that they satisfy the conditions (i), (ii) because $\tau(\mathfrak{n})$ is injective.

q.e.d.

Proposition 4.7. If $N$ is sufficiently large and if $x_{1}, \cdots, x_{N}$ are in sufficiently general position, then $\tau(\mathfrak{n})$ is an immersion for $\mathfrak{n}=\left(x_{1}, \cdots, x_{N}\right)$. 
Proof. Take $\mathfrak{n}=\left(x_{1}, \cdots, x_{N}\right)$ such that the conclusion of Lemma 4.6 holds for $\mathfrak{n}$. Then there exists a $G$-stable open subscheme $U_{0}$ of $H_{p, 2}^{N}$ such that $\tau(\mathfrak{n})\left(R_{0}\right) \subset U_{0}$ and $\tau(\mathfrak{n}): R_{0} \rightarrow U_{0}$ is proper. Thus $\tau(\mathfrak{n})_{*}\left(\mathcal{O}_{R_{0}}\right)$ is a coherent $\mathcal{O}_{U_{0}}$-module. Let $q$ be a $k$-rational point of $R_{0}$ and let $t=\tau(\mathfrak{n})(q)$. Since $\tau(\mathfrak{n})$ is injective, we get that $\left(\tau(\mathfrak{n})_{*}\left(\mathcal{O}_{R_{0}}\right)\right)_{t}=\mathcal{O}_{R_{0}, q} . \quad \mathcal{O}_{R_{0}, q}$ is therefore a finite $\mathcal{O}_{U_{0}, t}$-module. Thus we have only to show that the $d \tau(\mathfrak{n})_{q}$ of $T_{R_{0}, q}$ to $T_{H_{p, 2, t}^{N}}$ is injective, where $T_{R_{0}, q}$ (or, $T_{H_{p, 2}^{N}, t}^{N}$ ) is the Zariski tangent space of $R_{0}$ at $q$ (or, $H_{p, 2}^{N}$ at $t$, resp.). For if $d \tau(\mathfrak{n})_{q}$ is injective, that is, $\mathfrak{m}_{t} / \mathfrak{m}_{t}^{2} \rightarrow \mathfrak{m}_{q} / \mathfrak{m}_{q}^{2}$ is surjective, then by virtue of Nakayama's lemma on $\mathcal{O}_{R_{0}, q}$ we know that $\mathfrak{m}_{t} \mathcal{O}_{R_{0}, q}=\mathfrak{m}_{q}$. Then the facts that $\mathcal{O}_{U_{0}, t} / \mathfrak{m}_{t} \widetilde{\rightarrow}$ $\mathcal{O}_{R_{0}, q} / \mathfrak{m}_{q} \cong k$ and that $\mathcal{O}_{R_{0}, q}$ is a finite $\mathcal{O}_{U_{0}, t}$-module imply that $\mathcal{O}_{U_{0}, t} \rightarrow \mathcal{O}_{R_{0}, q}$ is surjective by Nakayama's lemma on $\mathcal{O}_{U_{0}, t}$. Now let us recall the results of Grothendieck on the Zariski tangent space of $R_{0}$ at $q$. Take the infinitesimal scheme $I=\operatorname{Spec}(k[\varepsilon]), \varepsilon^{2}=0$ and consider $T(q)=\{f \in$ $\left.\operatorname{Hom}_{k}\left(I, R_{0}\right) \mid f \cdot i=q\right\}$, where $i$ is the k-rational point of $I$. Then $T(q)$ can be naturally identified with $T_{R_{0}, q}$. For a given $f \in T(q)$ we get a locally free quotient $\mathcal{O}_{X \times k} I$-module $E_{f}$ of $\mathcal{O}_{X \times{ }_{k} I}^{\oplus p}$ such that for the natural morphism $g: X \rightarrow X \times_{k} I, g^{*}\left(E_{f}\right)=E_{f} / \varepsilon E_{f}=\tilde{F}_{q}$. Let us consider the following exact commutative diagram;

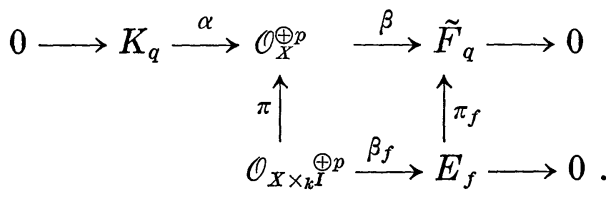

For a special element $f_{0}=q \cdot p$ of $T(q)$ ( $p$ is the structure morphism $I$ $\rightarrow \operatorname{Spec}(k))$, we get

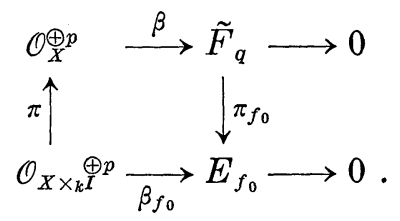

Pick an element $u$ of $K_{q}$, then there is an element $v$ of $\mathcal{O}_{X \times_{k} I}^{\oplus p}$ with $\alpha(u)=\pi(v)$. Since $\pi_{f} \cdot \beta_{f}(v)=\pi_{f_{0}} \cdot \beta_{f_{0}}(v)=\beta \cdot \alpha(u)=0, \beta_{f}(v)$ and $\beta_{f_{0}}(v)$ can be written in the forms $\varepsilon w$ and $\varepsilon w_{0}$ respectively with some $w \in E_{f}, w_{0}$ $\in E_{f_{0}}$. Hence we obtain an element $w^{\prime}=\pi_{f}(w)-\pi_{f_{0}}\left(w_{0}\right)$ of $F_{q}$ from $u$. It is easy to see that $w^{\prime}$ is uniquely determined by $u$ (i.e. independent of the choice of $v$ ) and that the map $u \stackrel{\theta(f)}{\longmapsto} w^{\prime}$ is an $\mathcal{O}_{X}$-homomorphism. 
By this way we get a map $\theta: T_{R_{0}, q}=T(q) \ni f \longmapsto \theta(f) \in \operatorname{Hom}_{o_{X}}\left(K_{q}, F_{q}\right)$. $\theta$ is an isomorphism as $k$-vector spaces (see [2] Proposition 5.1, Corollary 5.3). Let $0 \longrightarrow W_{t_{i}} \longrightarrow k^{\oplus p} \longrightarrow V_{t_{i}} \longrightarrow 0$ be the exact sequence of vector spaces corresponding to $t_{i} \in H_{p, 2}(k)$ with $t=\left(t_{1}, \cdots, t_{N}\right)$, then we get the following exact commutative diagram;

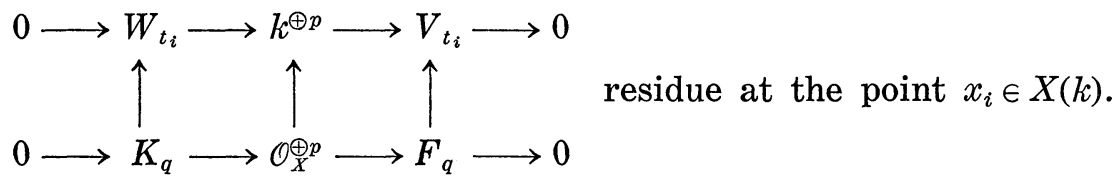

By a similar reason as above we know that $T_{H_{p, 2}, t_{i}}$ is isomorphic to $\operatorname{Hom}_{k}\left(W_{t_{i}}, V_{t_{i}}\right)$. Thus for an $f \in \operatorname{Hom}_{\odot_{X}}\left(K_{q}, F_{q}\right)=T_{R_{0}, q}$, we obtain an element $f\left(x_{i}\right)$ of $\operatorname{Hom}_{k}\left(W_{t_{i}}, V_{t_{i}}\right)=T_{H_{p, 2}, t_{i}}$ by taking the residue class of $f$ at $x_{i}$. The map $f \longmapsto\left(f\left(x_{1}\right), \cdots, f\left(x_{N}\right)\right)$ of $T_{R_{0}, q}$ to $T_{H_{p, 2}^{N}, t}$ is just $d \tau(\mathfrak{n})_{q}$. Then put $Z(\mathfrak{n})=\left\{q \in R_{0} \mid d \tau(\mathfrak{n})_{q}\right.$ is not injective $\}$ and $Z(\mathfrak{n})^{\prime}=$ $\operatorname{Supp}\left(\operatorname{coker}\left(\mathcal{O}_{H_{p, 2}^{N}}^{N} \rightarrow \tau(\mathfrak{n})_{*}\left(\mathcal{O}_{R_{0}}\right)\right)\right)$, then $Z(\mathfrak{n})=\tau(\mathfrak{n})^{-1}\left(Z(\mathfrak{n})^{\prime}\right)$ because $d \tau(\mathfrak{n})_{q}$ is injective if and only if $\mathcal{O}_{H_{p, 2}^{N}, t}^{N} \rightarrow\left(\tau(\mathfrak{n})_{*}\left(\mathcal{O}_{R_{0}}\right)\right)_{t}$ is surjective with $t=\tau(\mathfrak{n})(q)$ as was shown in the first part of this proof. Since $Z(\mathfrak{n})^{\prime}$ is closed in $H_{p, 2}^{N}$, so is $Z(\mathfrak{n})$ in $R_{0}$. For irreducible components $Z_{1}, \cdots, Z_{m}$ of $Z(\mathfrak{n})$, take a $k$-rational point $z_{i}$ of each $Z_{i}$. Let $S_{1,0} \subset \operatorname{Hom}_{\sigma_{X}}\left(K_{z_{1}}, \tilde{F}_{z_{1}}\right)$ be the kernel of the map $d \tau(\mathfrak{n})_{z_{1}}$. Since for a non-zero element $f \in S_{1,0}$, the set $\{x \in X \mid f(x)=0\}$ is closed, there exists a $k$-rational point $y_{1}$ of $X$ such that $f\left(y_{1}\right) \neq 0$. Then for $\mathfrak{n}_{1}=\left(x_{1}, \cdots, x_{N}, y_{1}\right)$, the kernel $S_{1,1}$ of $d \tau(\mathfrak{n})_{z_{1}}$ is a proper subspace of $S_{1,0}$. By induction on $\operatorname{dim}\left(\operatorname{ker}\left(d \tau(\mathfrak{n})_{z_{1}}\right)\right.$ ) we get a sequence of $k$-rational points $y_{1}, \cdots, y_{r_{1}}$ such that for $\mathfrak{n}_{r_{1}}=\left(x_{1}, \cdots, x_{N}, y_{1}\right.$, $\left.\cdots, y_{r_{1}}\right), d \tau\left(\mathfrak{n}_{r_{1}}\right)_{z_{1}}$ is injective. For the kernel $S_{2,0}$ of $d \tau\left(\mathfrak{n}_{r_{1}}\right)_{z_{2}}$ we obtain a sequence of $k$-rational points $y_{r_{1}+1}, \cdots, y_{r_{2}}$ such that $d \tau\left(\mathfrak{n}_{r_{2}}\right)_{z_{2}}$ is injective with $\mathfrak{n}_{r_{2}}=\left(x_{1}, \cdots, x_{N}, y_{1}, \cdots, y_{r_{2}}\right)$ by the same argument. Applying this argument to all $z_{i}$ 's repeatedly we get $\mathfrak{n}^{\prime}$ such that $d \tau\left(\mathfrak{n}^{\prime}\right)_{z_{i}}$ is injective for any $i$. Since $Z\left(\mathfrak{n}^{\prime}\right)$ contains none of $z_{1}, \cdots, z_{m}$, we know that $\operatorname{dim} Z\left(\mathfrak{n}^{\prime}\right)$ $<\operatorname{dim} Z(\mathfrak{n})$. By induction on the dimension of $Z(\mathfrak{n})$ we get $\tilde{\mathfrak{n}}$ such that $Z(\tilde{\mathfrak{n}})=\phi$.

q.e.d.

Let $\left(H_{p, r}^{N}\right)_{0}$ (or, $\left.\left(H_{p, r}^{N}\right)_{0}^{s}\right)$ be the set of points with 0-dimensional stabilizer group (or, properly stable points ([14] Definition 1.1), resp.) of $H_{p, r}^{N}$ with respect to the action of $P G L(p)$ and the linebundle which defines the Plücker coordinates. Then they are $P G L(p)$-stable open subsets of $H_{p, r}^{N}$ ([8] p. 10, [14] Theorem 3.1). By virtue of Proposition 4.3 and 
Proposition 4.7 we may assume that $R_{0}$ (or, $R_{s}$ ) is a subscheme of $H_{p, 2}^{N}$ (or, $\left(H_{p, 2}^{N}\right)_{0}^{s}$, resp.). Hence from now on we shall disregard $\tau(\mathfrak{n})$.

The following lemma is a general remark which seems well-known.

LEMMA 4.8. Let us consider the canonical action $\sigma$ of $P G L(p)$ on $\left(H_{p, r}^{N}\right)_{0}^{s}$. Then $\sigma$ is a free action ([8] Definition 0.8, (iv)).

Proof. Put $\psi=\left(\tilde{\sigma}, p_{2}\right): P G L(p) \times{ }_{k} H_{p, r}^{N} \rightarrow H_{p, r}^{N} \times{ }_{k} H_{p, r}^{N}$, where $\tilde{\sigma}$ is the canonical action of $P G L(p)$ on $H_{p, r}^{N}$ and $p_{2}$ is the projection $P G L(p)$ $\times_{k} H_{p, r}^{N} \rightarrow H_{p, r}^{N}$. We have to prove that $\psi_{0}=\left(\sigma, p_{2}\right): P G L(p) \times_{k}\left(H_{p, r}^{N}\right)_{0}^{s} \rightarrow$ $\left(H_{p, r}^{N}\right)_{0}^{s} \times_{k}\left(H_{p, r}^{N}\right)_{0}^{s}$ is a closed immersion. Take $k$-rational points $g, t=$ $\left(t_{1}, \cdots, t_{N}\right)$ of $P G L(p),\left(H_{p, r}^{N}\right)_{0}$ respectively. Then $t$ is represented by $N$-ple of $r \times p$ matrices $M(t)=\left(M_{1}(t), \cdots, M_{N}(t)\right)$ with $\operatorname{rank}\left(M_{i}(t)\right)=r$ $\left(M_{i}(t)\right.$ is not unique) and the action of $g=\left(g_{i j}\right)$ is given by the multiplication of matrices; $\left(M_{1}(t), \cdots, M_{N}(t)\right) \longmapsto\left(M_{1}(t)\left(g_{i j}\right), \cdots, M_{N}(t)\left(g_{i j}\right)\right)$. Let $V$ be the vector space over $k$ which consists of row vectors with length $p$, let $a_{1}^{(\ell)}, \ldots, a_{r}^{(\ell)}$ be the row vectors of $M_{\ell}(t)$ and let $g \alpha_{1}^{(\ell)}, \ldots, g \alpha_{r}^{(\ell)}$ be those of $M_{\ell}(t)\left(g_{i j}\right)$. Let $e_{1}, \cdots, e_{p-r}, \cdots, e_{p}$ be a basis of $V$ such that $e_{1} \wedge \cdots \wedge e_{p_{-r}} \wedge a_{1}^{(\ell)} \wedge \cdots \wedge a_{r}^{(\ell)} \neq 0$ and $e_{1} \wedge \cdots \wedge e_{p_{-r}} \wedge g a_{1}^{(\ell)} \wedge \cdots \wedge g a_{r}^{(\ell)}$ $\neq 0$ for any $1 \leq \ell \leq N$. Put $U_{\ell}=\left\{s \in H_{p, r}^{N} \mid s\right.$ is represented by a matrix with row vectors $b_{1}, \cdots, b_{r}\left(b_{i} \in V \times_{k} k(s)\right)$ such that $e_{1} \wedge \cdots \wedge e_{p-r} \wedge b_{1}$ $\left.\wedge \cdots \wedge b_{r} \neq 0\right\}$. Then $U_{\ell}$ is an affine open neighborhood of $t$ and $U_{\ell}$ $=\operatorname{Spec}\left(k\left[u_{i j}^{(\ell)}\right]\right), 1 \leq i \leq r, 1 \leq j \leq p-r$, that is, $U_{\ell} \stackrel{f_{\sim}^{(\ell)}}{\longrightarrow} \mathbf{A}_{k}^{r(p-r)}$. In fact if $s \in U_{\ell}$ is represented by row vectors $b_{1}, \cdots, b_{r}$ and if $b_{i}=\sum_{j=1}^{p} \alpha_{i j} e_{j}$, then the $r \times(p-r)$ matrix $B(s)^{-1} A(s)$ defines a point $s^{\prime}$ in $\mathbf{A}_{k}^{r(p-r)}$ and $f^{(\ell)}(s)=s^{\prime}$, where $B(s)=\left(\alpha_{i j} ; 1 \leq i \leq r, p-r+1 \leq j \leq p\right)$ and $A(s)$ $=\left(\alpha_{i j} ; 1 \leq i \leq r, 1 \leq j \leq p-r\right)$ (note that $\operatorname{det} B(s) \neq 0$ because of $e_{1}$ $\left.\wedge \cdots \wedge e_{p_{-r}} \wedge b_{1} \cdots \wedge b_{r}=(\operatorname{det} B(s)) e_{1} \wedge \cdots \wedge e_{p} \neq 0\right)$. Similarly we get an affine open neighborhood $V_{\ell}$ of $\sigma(g, t)$ which is also isomorphic to $\mathbf{A}_{k}^{r(p-r)}=\operatorname{Spec}\left(k\left[v_{i j}^{(\ell)}\right]\right), 1 \leq i \leq r, 1 \leq j \leq p-r$. Put $U=V_{1} \times_{k} \cdots \times_{k} V_{N}$ $\times_{k} U_{1} \times_{k} \cdots \times_{k} U_{N}$, then $U$ is an affine open neighborhood of $(\sigma(g, t), t)$ in $H_{p, r}^{N} \times_{k} H_{p, r}^{N}$. Let $x=\left(x_{i j}\right)$ be the system of homogeneous coordinates of $P G L(p)\left(P G L(p)\right.$ is the affine open set of $\mathbf{P}^{\left(p^{2}-1\right)} \operatorname{defined}$ by $\left.\operatorname{det}\left(x_{i j}\right) \neq 0\right)$. For $u^{(\ell)} \in U_{\ell}, x \in P G L(p), \sigma\left(x, u^{(\ell)}\right)$ is contained in $V_{\ell}$ if and only if $\operatorname{det}\left(B\left(\sigma\left(x, u^{(\ell)}\right)\right)=h_{\ell}\left(x, u^{(\ell)}\right) \neq 0\right.$. Thus $\psi^{-1}(U)=W$ is the affine open neighborhood of $(g, t)$ in $P G L(p) \times{ }_{k} U_{1} \times{ }_{k} \cdots \times{ }_{k} U_{N}$ defined by $\mathrm{h}_{1}\left(x, u^{(1)}\right)$ $\neq 0, \cdots, \mathrm{h}_{N}\left(x, u^{(N)}\right) \neq 0$. We may assume that $g_{11} \neq 0$ without loss of 
any generality. Take the affine open neighborhood $W^{\prime}$ of $(g, t)$ in $W$ defined by $x_{11} \neq 0$ and the system of inhomogeneous coordinates $\left(w_{i j}\right), w_{11}$ $=1$ of $P G L(p)$. Let us put

$$
C^{(\ell)}(u, w)=\left(\begin{array}{c}
u_{11}^{(1)}, \cdots, u_{1, p-r}^{(\ell)}, 1,0, \cdots, 0,0 \\
u_{21}^{(\ell)}, \cdots, u_{2, p-r}^{(\ell)}, 0,1,0, \cdots, 0 \\
\ldots \ldots, \\
u_{r 1}^{(\ell)}, \cdots, u_{r, p-r}^{(\ell)}, 0,0, \cdots, 0,1
\end{array}\right)\left(w_{i j}\right)
$$

and write $C^{(\ell)}(u, w)=\left(C_{1}^{(\ell)}, C_{2}^{(\ell)}\right)$, where $C_{1}^{(\ell)}$ is an $r \times(p-r)$ matrix and $C_{2}^{(\ell)}$ is an $r \times r$ matrix. Then for $\left(C_{2}^{(\ell)}\right)^{-1} C_{1}^{(\ell)}=\left(d_{i j}^{(\ell)}(u, w)\right)$, the map $\varphi: \Gamma\left(U, \mathcal{O}_{U}\right) \rightarrow \Gamma\left(W^{\prime}, \mathcal{O}_{W^{\prime}}\right)$ induced by $\psi$ is given by $\varphi\left(v_{i j}^{(\ell)}\right)=d_{i j}^{(\ell)}(u, w)$ and $\varphi\left(u_{i j}^{(\ell)}\right)=u_{i j}^{(\ell)}$. Consider the following equation;

$$
\left(\begin{array}{c}
C_{1}^{(1)} \\
\vdots \\
C_{1}^{(N)}
\end{array}\right)=\left(\begin{array}{ccc}
C_{2}^{(1)} & & 0 \\
& \ddots & \\
0 & C_{2}^{(N)}
\end{array}\right)\left(\begin{array}{c}
\left(v_{i j}^{(1)}\right) \\
\vdots \\
\left(v_{i j}^{(1)}\right)
\end{array}\right)
$$

The equation $\varphi(*)$ obtained from $(*)$ by replacing $v_{i j}^{(\ell)}$ by $\varphi\left(v_{i j}^{(\ell)}\right)$ is a linear equation with respect to $\left(w_{i j}\right)$ over the ring $\varphi\left(\Gamma\left(U, \mathcal{O}_{U}\right)\right)$ and the assumption that $t$ is contained in $\left(H_{p, r}^{N}\right)_{0}(k)$ implies that the solution of the equation $(*)$ is unique at the point $(\tilde{\sigma}(g, t), t)$. Thus there is a $\left(p^{2}-1\right)$ $\times\left(p^{2}-1\right)$ submatrix $L$ of the matrix of the linear equation $(*)$ such that for $\operatorname{det} L=F(u, v), F(u, v)$ is not zero at $(\tilde{\sigma}(g, t), t)$. Let $U^{\prime}$ be the affine open neighborhood of $(\tilde{\sigma}(g, t), t)$ in $U$ defined by $F(u, v) \neq 0$. Then we get that $w_{i j}=\varphi^{\prime}\left(F_{i j}(u, v)\right)$ with some $F_{i j}(u, v) \in \Gamma\left(U^{\prime}, \mathcal{O}_{U^{\prime}}\right)$ and $\varphi^{\prime}: \Gamma\left(U^{\prime}, \mathcal{O}_{U^{\prime}}\right) \rightarrow \Gamma\left(\psi^{-1}\left(U^{\prime}\right), \mathcal{O}_{W}\right)$ induced by $\psi$. Let $U_{0}$ be the affine open neighborhood of $(\tilde{\sigma}(g, t), t)$ in $U$ defined by

$$
\begin{aligned}
& F(u, v) \neq 0, \quad H_{\ell}(u, v)=h_{\ell}\left(F_{i j}(u, v), u^{(\ell)}\right) \neq 0, \\
& D(u, v)=\operatorname{det}\left(F_{i j}(u, v)\right) \neq 0 .
\end{aligned}
$$

Then $W_{0}=\psi^{-1}\left(U_{0}\right)$ is an affine open neighborhood of $(g, t)$ in $W$. Moreover, since $F(u, v)$ is invertible on $U_{0}$, a fortiori so is $\varphi\left(F(u, v)\right.$ ) on $W_{0}$, we know that $x_{11} \neq 0$ on $W_{0}$ (if $x_{11}=0$ at some point in $W_{0}$, then the constant terms of the equation $(*)$ is zero at that point and hence we have no solusions) and $\psi^{-1}(y)$ is one point for any $k$-rational point $y=\left(y_{1}, y_{2}\right)$ of $\psi\left(W_{0}\right)$. Thus $W_{0}$ is contained in $W^{\prime} \cap\left(P G L(p) \times_{k}\left(H_{p, r}^{N}\right)_{0}\right)$ because $\operatorname{dim} \psi^{-1}(y)=$ dimension of the stabilizer group of $y_{2}$. Now we get 


$$
\begin{aligned}
& R_{1}=\Gamma\left(U_{0}, \mathcal{O}_{U_{0}}\right)=k\left[u, v, F(u, v)^{-1}, H_{\ell}(u, v)^{-1}, D(u, v)^{-1}\right] \\
& R_{2}=\Gamma\left(W_{0}, \mathcal{O}_{W_{0}}\right)=k\left[u, w, \varphi(F(u, v))^{-1}, \varphi\left(H_{\ell}(u, v)\right)^{-1}, \varphi(D(u, v))^{-1}\right] .
\end{aligned}
$$

As was shown in the above argument, if $\varphi^{\prime \prime}: R_{1} \rightarrow R_{2}$ is the map induced by $\psi$, then $w_{i j}, u_{i j}^{(\ell)}$ are contained in the image of $\varphi^{\prime \prime}$, whence $\varphi^{\prime \prime}$ is surjective. Thus we get the following diagram;

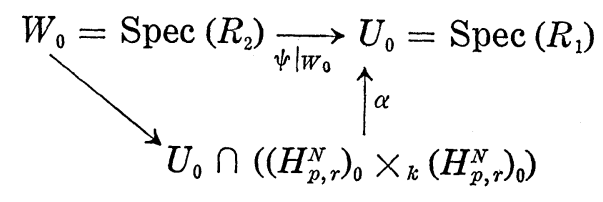

Since $\left.\psi\right|_{W_{0}}$ is a closed immersion and since the family of open sets such as $U_{0}$ covers $\psi\left(P G L(p) \times{ }_{k}\left(H_{p, r}^{N}\right)_{0}\right), \psi$ is an immersion on $P G L(p) \times{ }_{k}\left(H_{p, r}^{N}\right)_{0}$. Hence $\psi_{0}$ is also an immersion because $\left(H_{p, r}^{N}\right)_{0}^{s}$ is an open subscheme of $\left(H_{p, r}^{N}\right)_{0}$. On the other hand, we know that $\psi_{0}$ is a proper morphism by virtue of Proposition 3.2 of [14]. $\psi_{0}$ is therefore a closed immersion.

q.e.d.

The following lemma is an essential part in the proof of our main theorem.

LEMMA 4.9. Let $R_{s}$ and $G$ be as above. Then there exist a quasiprojective k-scheme $U$ and a morphism $\phi: R_{s} \rightarrow U$ such that $(U, \phi)$ is a geometric quotient with respect to the above action $\sigma$ of $G$ on $R_{s}$. Moreover $\phi: R_{s} \rightarrow U$ is a principal fibre bundle with group $G$ ([8] Proposition 0.9), in particular, $\phi$ is faithfully flat.

Proof. ${ }^{3)}$ Since $H_{p, 2}^{N}$ is projective and normal, $\left(H_{p, 2}^{N}\right)_{0}^{s}$ has a geometric quotient $(V, \psi)$ in the sense of Seshadrit) ([14] Theorem 7.2). Moreover $V$ is quasi-projective. Since $V$ is normal and since $\psi$ is equidimensional $\left(\operatorname{dim} G=\operatorname{dim} \psi^{-1}(v)\right.$ for every $\left.v \in V\right), \psi$ is universally open, a fortiori universally submersive by a criterion of Chevalley (E. G. A. Ch. IV, 14.4.4). Then $\psi:\left(H_{p, 2}^{N}\right)_{0}^{s} \rightarrow V$ is a principal fibre bundle with group $G$ ([8] Proposition 0.9). Now if we take $\mathfrak{n}=\left(x_{1}, \cdots, x_{N}\right)$ for which Lemma 4.6 and Proposition 4.7 hold, then there exists a $G$-stable open set $U_{s}$ of $H_{p, 2}^{N}$

3) Proposition 7.1 of [8], which made our proof of this lemma clear, was noticed the author by Professor T. Oda.

4) Seshadri assumed, in his definition of geometric quotients, only submersive. On the other hand, D. Mumford did "universally" submersive. 
in which $R_{s}$ is a closed subscheme. We may assume that $U_{s}$ is contained in $\left(H_{p, 2}^{N}\right)_{0}^{s}$ because so is $R_{s}$. Then $\psi\left(U_{s}\right)=V_{s}$ is an open subscheme and $\left.\psi\right|_{U_{s}}: U_{s} \rightarrow V_{s}$ is a principal fibre bundle with group $G$. In this situation we can apply Proposition 7.1 of [8] to $S=\operatorname{Spec}(k), X=R_{s}, Y=U_{s}$, $Q=V_{s}, \pi=\left.\psi\right|_{U_{s}}$ and to $L=\mathcal{O}_{R_{s}}$ and we get the required scheme $U=P$.

q.e.d.

Now we come to our main theorem of this section which implies the existence of coarse moduli schemes of stable vector bundles of rank 2 on a non-singular projective surface.

THEOREM 4.10. Let $X$ be a non-singular projective surface over $k$ and let $B\left(c_{1}, c_{2}\right)$ be the set of isomorphism classes of stable vector bundles on $X$ (with respect to a fixed very ample linebundle $H$ ) of rank 2 with fixed Chern classes $c_{1}, c_{2}$ (numerical equivalence). Then there exists $a$ quasi-projective algebraic $k$-scheme $M\left(c_{1}, c_{2}\right)$ satisfying the following conditions;

(i) There exists a bijective map $\phi$ of $B\left(c_{1}, c_{2}\right)$ to $M\left(c_{1}, c_{2}\right)(k)$.

(ii) Given an algebraic $k$-scheme $T$ and a locally free $\mathcal{O}_{X \times T}$-module $E$ such that $E_{t}$ is contained in $B\left(c_{1}, c_{2}\right)$ for any $t \in T(k)$, there exists $a$ morphism $f_{E}$ of $T$ to $M\left(c_{1}, c_{2}\right)$ with $f_{E}(t)=\varphi\left(E_{t}\right)$ for any $t \in T(k)$. Moreover, the correspondence $E \longmapsto f_{E}$ is functorial, that is, for a morphism $g: T^{\prime} \rightarrow T$ of algebraic k-schemes, $f_{E} \cdot g=f_{\left(1_{X} \times g\right) *(E)}$.

(iii) If an algebraic $k$-scheme $V$ and a map $\varphi^{\prime}: B\left(c_{1}, c_{2}\right) \rightarrow V(k)$ satisfy the above condition (ii), then there exists a unique morphism $\psi: M\left(c_{1}, c_{2}\right) \rightarrow V$ with $\psi(k) \cdot \varphi=\varphi^{\prime}, \psi \cdot f_{E}=f_{E^{\prime}}$, where $f_{E^{\prime}}$ is the morphism given by the condition (ii) for $V$.

Proof. We may assume that $B\left(c_{1}, c_{2}\right)$ satisfies the conditions (a), (b) before Proposition 4.3 for any $m \geq 0$ because the map $F \longmapsto F(m)$ of $B\left(c_{1}, c_{2}\right)$ to $B\left(c_{1}, c_{2}\right)(m)$ is bijective and because if $E$ is a locally free $\mathcal{O}_{X \times T^{-}}$ module such that $E_{t} \in B\left(c_{1}, c_{2}\right)$ for any $t \in T(k)$, then for $E(m)$ $=E \otimes p_{1}^{*}\left(H^{\otimes m}\right), E(m)_{t}$ is contained in $B\left(c_{1}, c_{2}\right)(m)$ for any $t \in T(k)$, where $p_{1}$ is the projection $X \times{ }_{k} T \rightarrow T$. Then we obtain a subscheme $R_{s}$ of $H_{p, 2}^{N}$, a locally free $\mathcal{O}_{X \times R_{s}}$-module $F$ and a surjective homomorphism $\alpha: \mathcal{O}_{X \times R s}^{\oplus p} \rightarrow F$. Moreover, $F$ has the following universal property; For any algebraic $k$-scheme $T$ and any surjective homomorphism of locally 
free $\mathcal{O}_{X \times T}$-modules $\beta: \mathcal{O}_{X \times T}^{\oplus p} \rightarrow E$ such that $E_{t} \in B\left(c_{1}, c_{2}\right)$ and $\beta_{t}$ induces an isomorphism $H^{0}\left(X, \mathcal{O}_{X}^{\oplus}\right) \widetilde{\rightarrow} H^{0}\left(X, E_{t}\right)$ for any $t \in T(k)$, there exists a unique morphism $h_{\beta}: T \rightarrow R_{s}$ such that $\left(1_{X} \times h_{\beta}\right)^{*}(F) \cong E$ and $\left(1_{X} \times h_{\beta}\right) *(\alpha)=\beta$. Now $R_{s}$ has a natural action $\sigma$ of $G=P G L(p)$ and by virtue of Lemma 4.9 there exists a geometric quotient $\left(M\left(c_{1}, c_{2}\right), \pi\right)$ of $R_{s}$ by $G$. We shall show that $M\left(c_{1}, c_{2}\right)$ is the desired algebraic $k$-scheme. As was shown in Lemma $4.9, M\left(c_{1}, c_{2}\right)$ is an algebraic $k$-scheme. $M\left(c_{1}, c_{2}\right)$ is quasi-projective. Since the set of orbits of $k$-rational points of $R_{s}$ is in bijective correspondence with $B\left(c_{1}, c_{2}\right)$ and since $M\left(c_{1}, c_{2}\right)$ is a geometric quotient of $R_{s}$ by $G$, we get a bijective map $\varphi$ of $B\left(c_{1}, c_{2}\right)$ to $M\left(c_{1}, c_{2}\right)(k)$. Assume that $T$ and $E$ satisfy the assumptions of (ii). Let $p_{2}$ be the projection of $X \times_{k} T$ to $T$ and let $\left(p_{2}\right)_{*}(E)=E^{\prime}$. Since $p_{2}$ is proper and flat and since $H^{1}\left(X \otimes_{k} k(t), E_{t}\right)=0$ for any $t \in T$ by our assumption $(a)$ put on $B\left(c_{1}, c_{2}\right)$, the natural map $\gamma_{t}: E^{\prime} \otimes_{\mathcal{O}_{T}} k(t) \rightarrow H^{0}\left(X \otimes_{k} k(t), E_{t}\right)$ is an isomorphism for any $t \in T$ ([10] p. 53, Corollary 3 ) and $E^{\prime}$ is a coherent $\mathcal{O}_{T}$-module. For a given $t \in T(k)$, take an affine open neighborhood $W_{0}$ and put $M=\Gamma\left(W_{0}, E^{\prime}\right)$. Let $m_{1}, \cdots, m_{p}$ be elements of $M$ such that their images by $M \rightarrow E^{\prime}$ $\otimes_{\mathcal{O}_{T}} k(t) \underset{r_{t}}{\sim} H^{0}\left(X, E_{t}\right)$ form a basis of $H^{0}\left(X, E_{t}\right)$. Then by Nakayama's lemma $m_{1}, \cdots, m_{p}$ generate $E^{\prime}$ at $t$. Thus there is an affine open neighborhood $W$ of $t$ such that the map $\left.\mathcal{O}_{W}^{\oplus p} \rightarrow E^{\prime}\right|_{W}$ given by $\left(a_{1}, \cdots, a_{p}\right)$ $\mapsto \sum_{i=1}^{p} a_{i} m_{i}$ is surjective. Then a natural map $\beta_{W}: \mathcal{O}_{X \times W}^{\oplus p}=p_{2}{ }^{*}\left(\mathcal{O}_{W}^{\oplus p}\right)$ $\left.\rightarrow p_{2}{ }^{*}\left(\left.E^{\prime}\right|_{W}\right) \rightarrow E\right|_{X \times W}$ is surjective because $E_{q}$ is generated by its global sections for any $q \in T$ (assumption $(a)$ ) and the map $H^{0}\left(X \otimes_{k} k(q), \mathcal{O}_{X \otimes k(q)}^{\oplus p}\right.$ ) $\rightarrow H^{0}\left(X \otimes_{k} k(q), E_{q}\right)$ induced by $\beta_{W}$ is bijective. Thus by the universal property of $\left(R_{s}, F\right)$ mentioned above we get a unique morphism $h_{W}\left(m_{1}\right.$, $\left.\cdots, m_{p}\right)$ of $W$ to $R_{s}$ with $\left.\left(1_{X} \times h_{W}\left(m_{1}, \cdots, m_{p}\right)\right) *(F) \cong E\right|_{X \times W}$ and $\left(1_{X}\right.$ $\left.\times h_{W}\left(m_{1}, \cdots, m_{p}\right)\right)^{*}(\alpha)=\beta_{W}$. Put $f_{W}=\pi \cdot h_{W}\left(m_{1}, \cdots, m_{p}\right)$. Then we claim that $f_{W}$ is independent of the choice of $m_{1}, \cdots, m_{p}$. For let $m_{1}^{\prime}, \cdots, m_{p}^{\prime}$ be another generators of $\left.E^{\prime}\right|_{W}$, then we get that $m_{j}=\sum_{j=1}^{p} m_{i}^{\prime} a_{i j}$ with some $a_{i j} \in \Gamma\left(W, \mathcal{O}_{W}\right)$. Then at any point $q$ of $W$, $\operatorname{det}\left(a_{i j}\right) \bmod \left(\mathfrak{m}_{q}\right)$ is not zero. Thus $\operatorname{det}\left(a_{i j}\right)$ is an invertible element of $\Gamma\left(W, \mathcal{O}_{W}\right)$, whence $\left(a_{i j}\right)$ is contained in $G L\left(p, \Gamma\left(W, \mathcal{O}_{W}\right)\right)$. Hence we get a $W$-valued point $a$ of $P G L(p, k)$. Then by the definition of the action of $G$ on $R_{s}$ we know that $\sigma(W)\left(a, h_{W}\left(m_{1}^{\prime}, \cdots, m_{p}^{\prime}\right)\right)=h_{W}\left(m_{1}, \cdots, m_{p}\right)$ as $W$-valued points of $R_{s}$. On the other hand, by the definition of a geometric quotient the following diagram is commutative; 


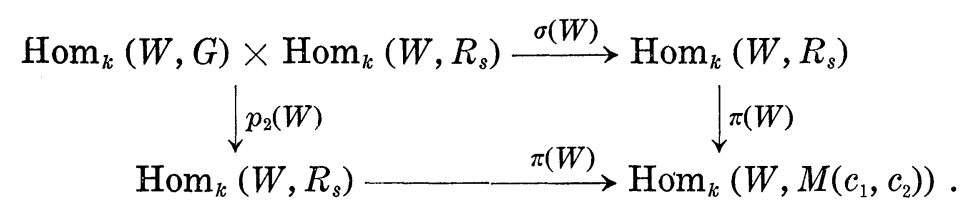

We get therefore that $\pi \cdot h_{W}\left(m_{1}^{\prime}, \cdots, m_{p}^{\prime}\right)=\left(\pi(W) \cdot p_{2}(W)\right)\left(a, h_{W}\left(m_{1}^{\prime}, \cdots, m_{p}^{\prime}\right)\right)$ $=(\pi(W) \cdot \sigma(W))\left(a, h_{W}\left(m_{1}^{\prime}, \cdots, m_{p}^{\prime}\right)\right)=\pi(W)\left(h_{W}\left(m_{1}, \cdots, m_{p}\right)\right)=\pi \cdot h_{W}\left(m_{1}, \cdots, m_{p}\right)$, which proves our claim. Let us cover $T$ by a family of such open sets $\left\{W_{\lambda}\right\}$ as $W$ above. Then there exists a family of morphisms $f_{\lambda}$ of $W_{\lambda}$ to $M\left(c_{1}, c_{2}\right)$. The above claim implies that $f_{\lambda}$ coincides with $f_{\mu}$ on $W_{\lambda}$ $\cap W_{\mu}$, whence we obtain a morphism $f_{E}$ of $T$ to $M\left(c_{1}, c_{2}\right)$ such that $\left.f_{E}\right|_{W_{\lambda}}=f_{\lambda}$. It is clear that $f_{E}(t)=\varphi\left(E_{t}\right)$ for any $t \in T(k)$. Next assume that a morphism of algebraic $k$-schemes $g: T^{\prime} \rightarrow T$ is given. For a $k$ rational point $t^{\prime}$ of $T^{\prime}$, take an affine open neighborhood $W$ of $g\left(t^{\prime}\right)$ and $m_{1}, \cdots, m_{p}$ as above. We have a natural map $\delta: g^{*}\left(E^{\prime}\right) \rightarrow\left(p_{2}^{\prime}\right)_{*}\left(1_{X} \times g\right)^{*}(E)$ $=E^{\prime \prime}$, where $p_{2}^{\prime}$ is the projection $X \times{ }_{k} T^{\prime} \rightarrow T^{\prime} . \delta\left(q^{\prime}\right)$ is surjective for any $q^{\prime} \in T^{\prime}(k)$ because for $q=g\left(q^{\prime}\right)$, the map $E^{\prime} \otimes_{{ }_{0}} k(q)=g^{*}\left(E^{\prime}\right) \otimes_{{ }_{0}^{\prime}} k\left(q^{\prime}\right)$ $\stackrel{\delta\left(q^{\prime}\right)}{\longrightarrow} E^{\prime \prime} \otimes_{\mathcal{o r}^{\prime}} k\left(q^{\prime}\right) \stackrel{\rightarrow}{\rightarrow} H^{0}\left(X,\left(1_{X} \times g\right)^{*}(E)_{q^{\prime}}\right)=H^{0}\left(X, E_{q}\right)$ is equal to $\gamma_{q}$ and $\gamma_{q}$ is an isomorphism. Thus $\delta$ is surjective, whence $\delta\left(g^{*}\left(m_{1}\right)\right), \cdots, \delta\left(g^{*}\left(m_{p}\right)\right)$ generate $E^{\prime \prime}$ on $g^{-1}(W)$. Hence if $\beta_{W}:\left.\mathcal{O}_{X \times W}^{\oplus p} \rightarrow E\right|_{X \times W}$ is the homomorphism defined by $m_{1}, \cdots, m_{p}$, then $\left(1_{X} \times g\right)^{*}\left(\beta_{W}\right):\left.\mathcal{O}_{X \times g-1}(W){ }^{\oplus P} \rightarrow\left(1_{X} \times g\right)^{*}(E)\right|_{X \times g^{-1}(W)}$ is that defined by $\delta\left(g^{*}\left(m_{1}\right)\right), \cdots, \delta\left(g^{*}\left(m_{p}\right)\right)$. Then we know that $h_{W}\left(m_{1}\right.$, $\left.\cdots, m_{p}\right) \cdot g=h_{g-1(W)}\left(\delta\left(g^{*}\left(m_{1}\right)\right), \cdots, \delta\left(g^{*}\left(m_{p}\right)\right)\right)$, that is, $f_{W} \cdot g=f_{g-1(W)}$. By the construction of $f_{E}$ we get that $f_{E} \cdot g=f_{\left(1_{X} \times g\right) *(E)}$. In order to prove (iii) let us consider the following diagram;

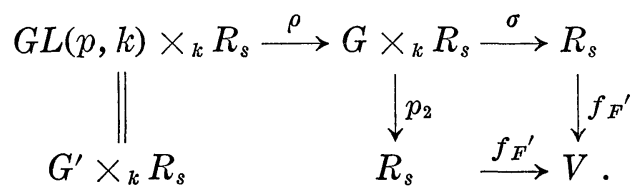

Let $e_{1}, \cdots, e_{p}$ be the fixed basis of $\mathcal{O}_{X \times R_{s}}^{\oplus p}$ and let $e_{1}^{\prime}, \cdots, e_{p}^{\prime}$ be the free basis of $\mathcal{O}_{X \times G^{\prime} \times R_{s}}^{\oplus p}$ corresponding to $e_{1}, \cdots, e_{p}$. The map $e_{j}^{\prime} \mapsto \sum_{i=1}^{p} e_{i}^{\prime} g_{i j}$ of $\mathcal{O}_{X \times\left(g_{i j}\right) \times R_{s}}^{\oplus p}$ to itself induces an automorphism $\theta$ of $\mathcal{O}_{X \times G^{\prime} \times R_{s}}^{\oplus p}$ and $\theta$ does an isomorphism of $\left(p_{2} \cdot \rho\right)^{*}(F)$ to $(\sigma \cdot \rho)^{*}(F)$. Thus we get that $f_{F^{\prime}} \cdot \sigma \cdot \rho$ $=f_{(\sigma \cdot \rho)^{*}\left(F^{\prime}\right)^{\prime}}=f_{\left(p_{2} \cdot \rho\right)^{*}(F)^{\prime}}=f_{F^{\prime}} \cdot p_{2} \cdot \rho$. Since $\rho$ is an epimorphism, we obtain that $f_{F^{\prime}} \cdot \sigma=f_{F^{\prime}} \cdot p_{2}$. Since $M\left(c_{1}, c_{2}\right)$ is a geometric quotient (a fortiori 
categorical quotient) of $R_{s}$ by $G$, there exists a unique morphism $\psi: M\left(c_{1}, c_{2}\right) \rightarrow V$ with $\psi \cdot f_{F}=f_{F^{\prime}}$. It is clear that $\psi(k) \cdot \varphi=\varphi^{\prime}$. Assume that an algebraic $k$-scheme $T$ and a locally free $\mathcal{O}_{X \times T}$-module $E$ satisfying the assumptions of (ii) are given. Let us cover $T$ by such a family of open sets $\left\{W_{\lambda}\right\}$ as in the above proof of (ii). Then there exists a family of morphisms $h_{\lambda}$ of $W_{\lambda}$ to $R_{s}$ such that $h_{\lambda}{ }^{*}\left(F^{\prime}\right)=\left.E\right|_{W_{\lambda}}$. Then by the functoriality of $f_{E}$ and $f_{E}^{\prime}$ we get

$$
\left.\psi \cdot f_{E}\right|_{W_{\lambda}}=\psi \cdot f_{h_{\lambda}^{*}(F)}^{*}=\psi \cdot f_{F^{\prime}} \cdot h_{\lambda}=f_{F}^{\prime} \cdot h_{\lambda}=\left.f_{E}^{\prime}\right|_{W_{\lambda}} .
$$

Thus we obtain that $\psi \cdot f_{E}=f_{E^{\prime}}$.

q.e.d.

Remark 4.12. Let $T$ be an algebraic $k$-scheme. Let $\mathscr{F}(T)$ be the set of isomorphism classes of locally free $\mathcal{O}_{X \times T}$-modules $E$ of rank $r$ such that for any $t \in T(k), E_{t}$ is a stable vector bundle of rank $r$ with Chern classes $c_{1}, c_{2}$. Consider an equivalence relation on $\mathscr{F}(T) ; E$ is equivalent to $E^{\prime}$ if and only if $E \cong E^{\prime} \otimes p_{2}{ }^{*}(L)$ for some linebundle $L$ on $T$. Let $\mathscr{V} \mathscr{B}_{X}\left(r, c_{1}, c_{2}\right)(T)$ be the quotient set of $\mathscr{F}(T)$ by this equivalence relation. For a morphism of algebraic $k$-schemes $f: T^{\prime} \rightarrow T$, if $E$ is contained in $\mathscr{F}(T)$, then $f^{*}(E)$ is a member of $\mathscr{F}\left(T^{\prime}\right)$ and if $E$ and $E^{\prime}$ are equivalent to each other, then so are $f^{*}(E)$ and $f^{*}\left(E^{\prime}\right)$. Thus we get a map $f^{*}: \mathscr{V} \mathscr{B}_{X}\left(r, c_{1}, c_{2}\right)(T) \rightarrow \mathscr{V} \mathscr{B}_{X}\left(r, c_{1}, c_{2}\right)\left(T^{\prime}\right)$. By this way $\mathscr{V} \mathscr{B}\left(r, c_{1}, c_{2}\right)$ is a contravariant functor from the category of algebraic $k$-schemes to the category of sets. Then the above theorem means that $M\left(c_{1}, c_{2}\right)$ is a coarse moduli scheme ([8] Definition 5.6) of $\mathscr{V}_{\mathscr{B}_{X}}\left(2, c_{1}, c_{2}\right)$ (note that $f_{E}$ $\left.=f_{E \otimes p_{2}^{*}(L)}\right)$.

Remark 4.12. Consider the following property $\left(^{*}\right)$ of a vector bundle $E$ on a non-singular projective surface $X$;

(*) If a locally free $\mathcal{O}_{X}$-submodule $F$ of $E$ is generated by its global sections outside a finite set of points of $X$, then

$$
h^{0}\left(F^{\prime}\right) / r(F)<h^{0}(E) / r(E) .
$$

Let $B\left(r, c_{1}, c_{2}\right)$ be the set of isomorphism classes of stable vector bundles of rank $r$ on $X$ with Chern classes $c_{1}, c_{2}$. Suppose the following is true;

(**) There exists an integer $m_{0}$ such that for any $m \geq m_{0}$ and any $E \in B\left(r, c_{1}, c_{2}\right), E(m)$ possesses the property (*). Then replacing Lemma 4.1 by $(* *)$, we can eliminate the assumption "rank 2 " in Theorem 4.10.

The following is a corollary to the proof of Theorem 4.10. 
COROLlaRY 4.10.1. $M\left(c_{1}, c_{2}\right)$ is non-singular (normal or Cohen-Macaulay) if and only if so is $R_{s}$.

Proof. Since $\pi: R_{s} \rightarrow M\left(c_{1}, c_{2}\right)$ is a principal fibre bundle with group $P G L(p), \pi$ is a smooth morphism. Then our assertion is clear by virtue of E. G. A. Ch IV, 6.3.4, 6.5.2 and 6.5.4.

q.e.d.

Remark 4.13. If $X$ is a non-singular projective curve, then the scheme corresponding to our $R_{s}$ is non-singular ([12] p. 324). Thus by the same reason as above we can prove that the coarse moduli schemes of stable vector bundles on $X$ are nonsingular (see [12] and the remark after Theorem 5 of [13]).

In general $M\left(c_{1}, c_{2}\right)$ is not necessary non-singular. In fact,

EXAMPLE 4.14. Let us construct such an example as $M\left(c_{1}, c_{2}\right)$ has singularities.

We shall begin with some general facts. Let $E$ be a simple vector bundle of rank $r$ on a non-singular projective surface $X$, let $E$ be generated by its global sections and let $N=h^{0}(E)$. Then we get an exact sequence

$$
0 \longrightarrow F \longrightarrow \mathcal{O}_{\bar{X}}^{\oplus N} \longrightarrow E \longrightarrow 0
$$

with some locally free $\mathcal{O}_{X}$-module $F$. By tensoring $E$ the dual sequence of the above, we get

$$
0 \longrightarrow E \otimes_{O_{X}} E^{\vee} \longrightarrow E^{\oplus N} \longrightarrow E \otimes_{O_{X}} F^{\vee} \longrightarrow 0 \text {. }
$$

Assume that $h^{1}(E)=h^{2}(E)=0$. Then we get the following exact sequence

$$
\begin{array}{r}
0 \longrightarrow H^{0}\left(X, E \otimes_{\mathscr{O}_{X}} E^{\vee}\right) \longrightarrow H^{0}(X, E)^{\oplus N} \longrightarrow H^{0}\left(X, E \otimes_{\mathcal{O}_{X}} F^{\vee}\right) \\
k \\
\longrightarrow H^{1}\left(X, E \otimes_{\mathcal{O}_{X}} E^{\vee}\right) \longrightarrow 0
\end{array}
$$

and $H^{1}\left(X, E \otimes_{\mathcal{O}_{X}} F^{\vee}\right) \widetilde{\rightarrow} H^{2}\left(X, E \otimes_{\mathcal{O}_{X}} E^{\vee}\right)$. Let $x$ be the $k$-rational point of $Q=$ Qut $_{0_{X} / k}^{\oplus N}$ which corresponds to the above surjective homomorphism $\mathcal{O}_{X}^{\oplus N} \rightarrow E$. Then the Zariski tangent space $T_{Q, x}$ of $Q$ at $x$ is isomorphic to $H^{0}\left(X, E \otimes_{O_{X}} F^{\vee}\right.$ ) (see the proof of Proposition 4.7). Now the above exact sequence implies

1) $\operatorname{dim} T_{Q, x}=h^{0}\left(E \otimes_{O_{X}} F^{\vee}\right)=h^{1}\left(E \otimes_{O_{X}} E^{\vee}\right)+N^{2}-1$. 
On the other hand, by virtue of Riemann-Roch theorem

$$
\text { 2) } \begin{aligned}
h^{1}\left(E \otimes_{O_{X}} E^{\vee}\right)= & 1+h^{2}\left(E \otimes_{\mathcal{O}_{X}} E^{\vee}\right)+(r-1)\left(c_{1}(E), c_{1}(E)\right)-2 r c_{2}(E) \\
& +r^{2} \chi\left(\mathcal{O}_{X}\right) .
\end{aligned}
$$

Thus in order to compute $h^{0}\left(E \otimes_{\mathcal{O}_{X}} F^{\vee}\right)$ we have only to do $h^{2}\left(E \otimes_{\mathcal{O}_{X}} E^{\vee}\right)$ $=h^{0}\left(E \otimes_{\mathcal{O}_{X}} E^{\vee} \otimes_{\mathcal{O}_{X}} K_{X}\right)$, where $K_{X}$ is the canonical bundle on $X$. Next assume in addition to the above that rank of $E$ is 2 .

Then we get a canonical exact sequence

$$
\begin{gathered}
0 \longrightarrow \mathcal{O}_{X} \stackrel{i}{\longrightarrow} E \otimes_{\mathscr{O}_{X}} E^{\vee} \longrightarrow S^{2}(E) \otimes_{O_{X}} \operatorname{det}\left(E^{\vee}\right) \longrightarrow 0, \\
{\mathscr{E} n d_{O_{X}}}(E)
\end{gathered}
$$

where $\mathcal{O}_{X}$ is contained in $\mathscr{E}_{n} d_{O_{X}}(E)$ as scalar multiplications. Moreover if the characteristic $p$ of $k$ is not equal to 2 , then we get that $(\operatorname{Tr} / 2) \cdot i$ $=i d$, where $\operatorname{Tr}$ is the trace map of $\mathscr{E}_{n \mathscr{O}_{X}}(E)$ to $\mathcal{O}_{X}$. The above sequence therefore splits under the assumption $p \neq 2$. Let $\mathcal{O}_{\mathbf{P}(E)}(1)$ be the tautological linebundle of $E$, then $S^{2}(E)=\pi_{*}\left(\mathcal{O}_{\mathbf{P}(E)}(2)\right)$, where $\pi$ is the natural projection of $\mathbf{P}(E)$ to $X$. Thus

$$
\text { 3) } \begin{gathered}
h^{0}\left(E \otimes_{\mathcal{O}_{X}} E^{\vee} \otimes_{\mathcal{O}_{X}} K_{X}\right)=p_{g}+h^{0}\left(S^{2}(E) \otimes_{O_{X}} \operatorname{det}\left(E^{\vee}\right) \otimes_{O_{X}} K_{X}\right) \\
=p_{g}+h^{0}\left(\mathcal{O}_{\mathbf{P}(E)}(2) \otimes_{O_{\mathbf{P}}(E)} \pi^{*}\left(\operatorname{det}\left(E^{\vee}\right) \otimes_{O_{X}} K_{X}\right)\right)
\end{gathered}
$$

under the assumption that $p \neq 2$ or $h^{1}\left(K_{X}\right)=0$.

Now let us construct an irreducible algebraic $k$-scheme $U$ and a vector bundle $F$ of rank 2 on $X \times_{k} U$ such that $h^{0}\left(F_{x} \otimes_{O_{X}} F_{x}^{\vee} \otimes_{O_{X}} K_{X}\right), x$ $\in U(k)$ is not constant and that $F_{x}$ is stable for any $x \in U(k)$. Note first of all that $M=M\left(c_{1}\left(F_{x}\right), c_{2}\left(F_{x}\right)\right)(x \in U(k))$ has singularities. For by virtue of Theorem 4.10 , (ii) there exists a morphism $f_{F}: U \rightarrow M$ such that $f_{F}(x)=\varphi\left(F_{x}\right)$ for any $x \in U(k)$. We may assume that for all $m \geq 0$, $B\left(c_{1}\left(F_{x}\right), c_{2}\left(F_{x}\right)\right)(m)$ satisfies the conditions (a) and (b) before Proposition 4.3 (see remarks at the beginning of the proof of Theorem 4.10). Then we get a principal fibre bundle $\psi: R_{s} \rightarrow M$ with group $G=P G L(N)$ $\left(N=h^{0}\left(F_{x}\right)\right)$. The above argument shows that for $x \in U(k), y \in$ $\psi^{-1}\left(f_{F}(x)\right)(k), T_{R_{s}, y} \cong H^{0}\left(X, F^{\prime \vee} \otimes_{O_{X}} F_{x}\right)$, where $F^{\prime}$ is the kernel of a canonical homomorphism $\mathcal{O}_{X}^{\oplus N} \rightarrow F_{x}$. Thus $\operatorname{dim} T_{M, f_{F}(x)}=\operatorname{dim} T_{R_{s}, y}-\operatorname{dim} G$ $=h^{0}\left(F^{\prime \vee} \otimes_{O_{X}} F_{x}\right)-N^{2}+1=h^{1}\left(F_{x} \otimes_{O_{X}} F_{x}^{\vee}\right)$ by formula (1). Since $h^{0}\left(F_{x}\right.$ $\left.\otimes_{O_{X}} F_{x}^{\vee} \otimes_{O_{X}} K_{X}\right)=h^{2}\left(F_{x} \otimes_{O_{X}} F_{x}^{\vee}\right)$ is not constant, so is $h^{1}\left(F_{x} \otimes_{O_{X}} F_{x}^{\vee}\right)$ by formula (2). Thus $\operatorname{dim} T_{M, f_{F}(x)}$ is not constant. On the other hand, 
since $U$ is irreducible, so is $f_{F}(U)$, whence $f_{F}(U)$ is contained in a conected component of $M$. We know therefore that $M$ has singularities.

To construct $(U, F)$, assume that (i) $\mathrm{Pic}(X) \cong Z$ as a group, (ii) the complete linear system $\left|K_{X}\right|$ of the canonical bundle $K_{X}$ of $X$ has no base points, (iii) there exists a non-singular irreducible curve $T$ on $X$ with $(K, T) \geq(T, T)+5$, where $K$ is a member of $\left|K_{X}\right|$. Pick a curve $T$ of genus $g$ on $X$ satisfying the condition (iii). Let $D$ be a divisor on $T$ with $\operatorname{deg} D=(K, T)=t$. Take a regular vector bundle $E$ in $R^{2}(X, T, D)$ ([6] p. 109, p. 112). Let $E$ be defined by $Y$ and let $Y^{\prime}$ be the center of $\left(\operatorname{elm}_{Y}^{0}\right)^{-1}$. We have to compute the dimension of the linear system $\mathscr{L}=$ $\left|\mathcal{O}_{\mathbf{P}(E)}(2) \otimes_{\mathcal{O P}(E)} \pi^{*}\left(\operatorname{det}\left(E^{\vee}\right) \otimes_{\mathcal{O}_{X}} K_{X}\right)\right| . \quad$ Pick a member $Z^{\prime}$ of $L$ and write $Z^{\prime}=A^{\prime}+\alpha \pi^{-1}(T) \quad\left(A^{\prime} \not \supset \pi^{-1}(T), \alpha \geq 0\right), A^{\prime} \cdot \pi^{-1}(T)=n Y^{\prime}+B^{\prime} \quad\left(B^{\prime} \not \supset Y^{\prime}\right)$. Let $A$ be the proper transform of $A^{\prime}$ by elm $Y^{\prime}, H$ be the divisor (0) $\times X$ of $\mathbf{P}^{1} \times{ }_{k} X, \pi_{0}$ be the projection $\mathbf{P}^{1} \times{ }_{k} X \rightarrow X$ and let $C=H \cdot \pi_{0}^{-1}(T)$. Then by virtue of Appendix II we get the following cases;

I) The case where $n=0$ : Then $A \sim 2 H+(1-\alpha) \pi_{0}^{-1}(T)+\pi_{0}^{-1}(K)$ and $A \cdot \pi_{0}^{-1}(T) \supset 2 Y$. Thus $A \cdot \pi_{0}^{-1}(T) \sim 2 C+(1-\alpha)\left(\pi_{0}\right)_{T}^{-1}(T \cdot T)+\left(\pi_{0}\right)_{T}^{-1}(K \cdot T)$ and $A \cdot \pi_{0}^{-1}(T)=2 Y+\left(\pi_{0}\right)_{T}^{-1}(B) \sim 2 C+\left(\pi_{0}\right)_{T}^{-1}(2 D+B)$ with some positive divisor $B$ on $T$. Hence we have that $2 D-K \cdot T \sim(1-\alpha)(T \cdot T)-B$. Comparing the degrees of both sides, we get that $t=(1-\alpha)(T, T)$ - $\operatorname{deg} B \leq(1-\alpha)(T, T)$, which is impossible because of the assumptions (i) and (iii).

II) The case where $n=1$ : Then $A \sim 2 H-\alpha \pi_{0}^{-1}(T)+\pi_{0}^{-1}(K), A \cdot \pi_{0}^{-1}(T)$ $\supset Y$ and $A \cdot \pi_{0}^{-1}(T) \not \supset 2 Y$. Thus $A \cdot \pi_{0}^{-1}(T) \sim 2 C-\alpha\left(\pi_{0}\right)_{T}^{-1}(T \cdot T)+\left(\pi_{0}\right)_{T}^{-1}(K \cdot T)$ and $A \cdot \pi_{0}^{-1}(T) \sim Y+C+\left(\pi_{0}\right)_{T}^{-1}(B) \sim 2 C+\left(\pi_{0}\right)_{T}^{-1}(D+B)$ with some positive divisor $B$ on $T$. Hence we have that $D-K \cdot T \sim-\alpha(T \cdot T)-B$. Since the degree of the left hand side is zero, we know that $B=0$ and $\alpha=0$. Thus $A \cdot \pi_{0}^{-1}(T)=Y+C^{\prime}$ with some $C^{\prime}=P \times T, P \in \mathbf{P}_{k}^{1}$ and $A$ is a member of $\left|2 H+\pi_{0}^{-1}(K)\right|$. Therefore choosing a suitable system of homogeneous coordinates $X_{0}, X_{1}$ of $\mathbf{P}_{X}^{1}, C^{\prime}$ is defined by $\bar{X}_{1}=0$ with the restriction $\bar{X}_{i}$ of $X_{i}$ to $\mathbf{P}_{T}^{1}$ and $A$ is defined by $s_{1} X_{0}^{2}+s_{2} X_{0} X_{1}+s_{3} X_{1}^{2}=0$ with some $s_{1}, s_{2}, s_{3}$ in $H^{0}\left(X, K_{X}\right)$. Since $A \cdot \pi_{0}^{-1}(T)=Y+C^{\prime}$, we know that $\bar{s}_{3}=0, s_{1}$ and $s_{2}$ have no common zeros on $T$ and $Y$ is defined by $\bar{s}_{1} \bar{X}_{0}+\bar{s}_{2} \bar{X}_{1}=0$, where $\bar{s}_{i}$ is the restriction of $s_{i}$ to $T$. Conversely pick three elements $s_{1}, s_{2}, s_{3}$ of $H^{0}\left(X, K_{X}\right)$ such that $s_{1}$ and $s_{2}$ have no common zeros on $T$ and $\bar{s}_{3}=0$ (such $s_{1}, s_{2}, s_{3}$ exist because of the assumption (ii)) and define $A$ (or, $Y$ ) by $s_{1} X_{0}^{2}+s_{2} X_{0} X_{1}+s_{3} X_{1}^{2}=0$ (or, $\bar{s}_{1} \bar{X}_{0}+\bar{s}_{2} \bar{X}_{1}=0$, resp.). Then 
$A \sim 2 H+\pi_{0}^{-1}(K), A \supset Y$ and $A \not \supset 2 Y$. Hence the regular vector bundle $E$ defined by $Y$ is contained in $R^{2}(X, T, K \cdot T)$, the proper transform of $A$ by $\operatorname{elm}_{Y}^{0}$ is a member of $\mathscr{L}$ for $E$ and $n=1$. We know therefore that this case occurs if and only if $E$ is defined by $\left(\bar{s}_{1}, \bar{s}_{2}\right)$ with some $s_{1}, s_{2} \in H^{0}\left(X, K_{X}\right)$ (see [6] Principle 2.6).

III) The case where $n=2$ and Sing $\left(A^{\prime}\right) \supset Y^{\prime}$ : Then $A+\alpha \pi_{0}^{-1}(T)$ $\sim 2 H-\pi_{0}^{-1}(T)+\pi_{0}^{-1}(K)$ and $A \cdot \pi_{0}^{-1}(T) \not \supset Y$. Let $Z$ be a member of $\left|2 H-\pi_{0}^{-1}(T)+\pi_{0}^{-1}(K)\right|$ and assume that $Z$ contains $Y$. Then $Z \cdot \pi_{0}^{-1}(T)$ $\sim 2 C+\left(\pi_{0}\right)_{T}^{-1}(K \cdot T)-\left(\pi_{0}\right)_{T}^{-1}(T \cdot T)$ and $Z \cdot \pi_{0}^{-1}(T) \sim Y+C+\left(\pi_{0}\right)_{T}^{-1}(B) \sim 2 C+$ $\left(\pi_{0}\right)_{T}^{-1}(D+B)$ with some positive divisor $B$ on $T$. Thus $0=\operatorname{deg}(K \cdot T)$ $-\operatorname{deg} D=\operatorname{deg} B+\operatorname{deg}(T \cdot T)>0$, which is a contradiction. Hence the set of members $Z^{\prime}$ of $L$ satisfying $n=2$ and $\operatorname{Sing}\left(Z^{\prime}\right) \supset Y^{\prime}$ is just that of total transforms of members of $\left|2 H-\pi_{0}^{-1}(T)+\pi_{0}^{-1}(K)\right|$ by $\operatorname{elm}_{Y}^{0}$.

IV) The case where $n=2$ and Sing $\left(A^{\prime}\right) \not \supset Y^{\prime}$ : Then $A \sim 2 H-\pi_{0}^{-1}(T)$ $+\pi_{0}^{-1}(K)$ and $A \cdot \pi_{0}^{-1}(T) \supset 2 Y$. We know by the same reason as in (I) that this case can not occur either.

Combining the results from (I) to (IV), we know

(A) if $D \in|K \cdot T|$ and if $E$ is defined by $\left(\bar{s}_{1}, \bar{s}_{2}\right)$ with some $s_{1}, s_{2}$ $\in H^{0}\left(X, K_{X}\right)$, then $h^{0}\left(E \otimes_{O_{X}} E^{\vee} \otimes_{O_{X}} K_{X}\right)=h^{0}\left(K_{X}\right)+h^{0}\left(S^{2}(E) \otimes_{O_{X}} \operatorname{det}\left(E^{\vee}\right)\right.$ $\left.\otimes_{\mathcal{O}_{X}} K_{X}\right) \geq p_{g}+\operatorname{dim}\left|2 H+\pi_{0}^{-1}(K)-\pi_{0}^{-1}(T)\right|+2=p_{g}+3 h^{0}\left(\mathcal{O}_{X}(-T) \otimes_{\mathcal{O}_{X}} K_{X}\right)$ +1 ,

(B) otherwise $h^{0}\left(E \otimes_{\mathcal{O}_{X}} E^{\vee} \otimes_{\mathcal{O}_{X}} K_{X}\right)=p_{g}+3 h^{0}\left(\mathcal{O}_{X}(-T) \otimes_{\mathcal{O}_{X}} K_{X}\right)$, under the assumption that $p \neq 2$ or $h^{1}\left(K_{X}\right)=0$ by virtue of formula (3).

Next put $V=\left\{Y \mid Y\right.$ is a section of $\left(\pi_{0}\right)_{T}: \mathbf{P}_{T}^{1} \rightarrow T$ such that $Y \in \mid C$ $+\left(\pi_{0}\right)_{T}^{-1}(D) \mid$ with some divisor $D$ on $T$ whose degree is $\left.t\right\}$, then $V$ is an open subscheme of a union of finite number of connected components of $\operatorname{Hilb}_{\mathbf{P}_{T}^{1} / k}$. Let $\tilde{Y}$ be the universal family on $\mathbf{P}_{T}^{1} \times_{k} V$ induced from that on $\mathbf{P}_{T}^{1} \times_{k} \operatorname{Hilb}_{\mathbf{P}_{T}^{1 / k}}$. Let $s_{1}, s_{2}$ be elements of $H^{0}\left(X, K_{X}\right)$ such that they have no common zeros on $T$ (see the assumption (ii)) and define $Y_{0}$ by $\bar{s}_{1} \bar{X}_{0}$ $+\bar{s}_{2} \bar{X}_{1}=0$, then $Y_{0}$ corresponds to a $k$-rational point $y_{0}$ of $V$. Since for the normal bundle $N_{Y_{0} / \mathbf{P}_{T}^{1}}$ of $Y_{0}$ in $\mathbf{P}_{T}^{1}, \operatorname{deg}\left(N_{Y_{0} / \mathbf{P}_{T}^{1}}\right)=2(K, T)=2 t$ $>2(((T, T)+(K, T)) / 2+2)=2(g+1)$, we have that $H^{1}\left(Y_{0}, N_{Y_{0} / \mathbf{P}_{T}^{1}}\right)=0$, whence $V$ is smooth at $Y_{0}$ and $\operatorname{dim} V=h^{0}\left(N_{Y_{0} / \mathbf{P}_{T}^{1}}\right)=2 t-g+1$ at $y_{0}$. (see [2] Corollary 5.4). On the other hand, for the natural morphism $h$ of $V$ to the Jacobian variety $J$ of $T$, since $h^{-1}(D)$ is an open subset of $\left|C+\pi_{0}^{-1}(D)\right|$, we get that $\operatorname{dim} h^{-1}(K \cdot T)=2 h^{0}\left(K_{X} \otimes_{O_{X}} \mathcal{O}_{T}\right) \leq t+2$ by 
Clifford's theorem (note that $K \cdot T$ is a special divisor on $T$ ). Thus if $U$ is the irreducible component of $V$ containing $y_{0}$, then $\operatorname{dim} U=2 t-g$ $+1>t+2 \geq \operatorname{dim} h^{-1}(K \cdot T)$, which implies that $U \supseteq h^{-1}(K \cdot T)$. Moreover we can construct a vector bundle $F$ on $X \times_{k} U$ such that for any $y \in U(k), F_{y}$ is the regular vector bundle defined by $\tilde{Y}_{y}$ ([6] Theorem 2.9). For any $y \in U(k), c_{1}\left(F_{y}\right)=T$ and $c_{2}\left(F_{y}\right)=t$ and $F_{y}$ is simple because $(T, T)<t$ ([6] Corollary 3.10.1). By virtue of Proposition A.1 and the assumption (i), we know that for every $y \in U(k), F_{y}$ is a stable vector bundle. On the other hand, $h^{0}\left(F_{y_{0}} \otimes_{\mathcal{O}_{X}} F_{y_{0}}^{\vee} \otimes_{\mathcal{O}_{X}} K_{X}\right) \geq p_{g}+3 h^{0}\left(\mathcal{O}_{X}(-T) \otimes_{\mathcal{O}_{X}} K_{X}\right)$ $+1=\beta+1$ by virtue of (A) and if a $k$-rational point $y$ of $U$ is not contained in $h^{-1}(K \cdot T)(k)$, then $h^{0}\left(F_{y} \otimes_{\mathcal{O}_{X}} F_{y}^{\vee} \otimes_{\mathcal{O}_{X}} K_{X}\right)=\beta$ by virtue of (B).

Finally we have to show that there exist $X$ and $T$ which satisfy the conditions (i), (ii) and (iii). For instance let $X$ be a general hypersurface of degree $n(n \geq 6)$ in $\mathbf{P}_{k}^{3}$, and let $T=S \cdot X$, where $S$ is a general hypersurface of degree $m(1 \leq m \leq n-5)$ in $\mathbf{P}_{k}^{3}$. Then it is well-known that $X$ and $T$ satisfy the conditions (i), (ii) and (iii) (note that $h^{1}\left(K_{X}\right)$ $=0)$.

All the moduli schemes are smooth under a suitable assumption on their base space. In fact

Proposition 4.15. Let $X$ be a non-singular projective surface such that for the canonical bundle $K_{X}$ of $X, K_{X} \neq \mathcal{O}_{X}$ and $\left|-K_{X}\right| \neq \phi$. Then $M\left(c_{1}, c_{2}\right)$ on $X$ is smooth for any numerical equivalence classes $c_{1}, c_{2}$.

Proof. We may assume that for any $m \geq 0, B\left(c_{1}, c_{2}\right)(m)$ satisfies the conditions (a) and (b) before Proposition 4.3. Then we can construct the subscheme $R_{s}$ of $H_{N, 2}^{r}$ for $B\left(c_{1}, c_{2}\right)$. By virtue of Corollary 4.11.1 we have only to show that $R_{s}$ is smooth. Take an arbitrary $k$-rational point $x$ of $R_{s}$, then $x$ corresponds to an exact sequence

$$
0 \longrightarrow F \longrightarrow \mathcal{O}_{X}^{\oplus N} \longrightarrow E \longrightarrow 0
$$

with some $E \in B\left(c_{1}, c_{2}\right)$. Since $B\left(c_{1}, c_{2}\right)$ satisfies the condition $(a), H^{1}(X, E$ $\left.\otimes_{O_{X}} F^{\vee}\right) \cong H^{2}\left(X, E \otimes_{O_{X}} E^{\vee}\right)$ as in Example 4.14. If $H^{2}\left(X, E \otimes_{O_{X}} E^{\vee}\right) \neq 0$, then $H^{0}\left(X, E \otimes_{\mathcal{O}_{X}} E^{\vee} \otimes_{\mathcal{O}_{X}} K_{X}\right) \neq 0$ by Serre duality. On the other hand, our assumption on $K_{X}$ implies that a global section of $E \otimes_{O_{X}} E^{\vee} \otimes_{0_{X}} K_{X}$ provides that of $E \otimes_{O_{X}} E^{\vee} \otimes_{O_{X}} K_{X} \otimes_{O_{X}} K_{X}^{\vee} \cong E \otimes_{O_{X}} E^{\vee}$ which has zeros. But this is impossible because $H^{0}\left(X, E \otimes_{\mathcal{O}_{X}} E^{\vee}\right)=\operatorname{End}_{\mathcal{O}_{X}}(E)$ consists of multiplications of constants (i.e. $E$ is simple). Thus we know that 
$H^{2}\left(X, E \otimes_{O_{X}} E^{\vee}\right)=0$, whence $\quad H^{1}\left(X, E \otimes_{O_{X}} F^{\vee}\right)=0$. Let $S=\operatorname{Spec}(A)$, $\bar{S}=\operatorname{Spec}(A / I)$ with some finite dimensional local $k$-algebra $A$ and an ideal $I$ of $A$ and let $j: X \rightarrow X \times_{k} \bar{S}=X_{\bar{S}}, h: X_{\bar{S}} \rightarrow X \times_{k} S=X_{S}$ be natural closed immersions. Assume that there exists an exact sequence of locally free $\mathcal{O}_{X \bar{S}}$-modules

$* * *)$

$$
0 \longrightarrow F(\bar{S}) \longrightarrow \mathcal{O}_{\bar{X} \bar{S}}^{\oplus N} \longrightarrow E(\bar{S}) \longrightarrow 0
$$

whose inverse image by $j$ is $(* *)$. We have to lift this sequence to $X_{S}$. In order to do this, using an induction on $\operatorname{dim}_{k} A$, we can reduce to the case where $\operatorname{dim}_{k} I=1$ because there exists an ideal $J$ of $A$ with $\operatorname{dim}_{k} J=1$ and $J \subset I$. Since $E(\bar{S})$ is locally free, we can lift locally (***) to $X_{S}$. Thus there exists a class of obstruction for lifting in $H^{1}(X, E(\bar{S})$ $\otimes_{\sigma_{X \bar{S}}} F(\bar{S})^{\vee} \otimes_{\sigma_{\bar{S}}} I$ ) (see [2] Corollary 5.2). From the assumption that $\operatorname{dim}_{k} I=1$, we know that $E(\bar{S}) \otimes_{o_{\bar{X} \bar{S}}} F(\bar{S})^{\vee} \otimes_{o_{\bar{S}}} I \cong E \otimes_{o_{X X}} F^{\vee}$. Since $H^{1}\left(X, E \otimes_{O_{X}} F^{\vee}\right)=0, H^{1}\left(X, E(\bar{S}) \otimes_{o_{X \bar{S}}} F(\bar{S})^{\vee} \otimes_{o_{\bar{S}}} I\right)=0$, which implies that there exists an exact sequence of locally free $\mathcal{O}_{X_{S}}$-modules

$$
0 \longrightarrow F(S) \longrightarrow \mathcal{O}_{X_{S}}^{\oplus N} \longrightarrow E(S) \longrightarrow 0
$$

whose inverse image by $h$ is $(* * *)$. Therefore $R_{s}$ is smooth at $x$ (see [9] Lecture 22).

q.e.d.

\section{Appendix.}

I. Let us prove the following proposition which is a corollary to Theorem 1.12 and Theorem 3.10 of [6].

Proposition A.1. Let $X$ be a non-singular projective variety over $k$ with $\operatorname{dim} X=2$ or 3 and $\operatorname{Pic}(X) \cong Z$ as an abstract group. Then $a$ vector bundle $E$ of rank 2 on $X$ is stable if and only if $E$ is simple.

Proof. The "only if" part was shown in [15]. Let us prove that if $E$ is not stable, then $E$ is not simple. Let $H$ be a linebundle on $X$ which is a generator of $\operatorname{Pic}(X)$ (we may assume that $H$ is ample). Since $E(n)=E \otimes_{{ }_{0 X}} H^{\otimes n}$ is simple (or stable) if and only if so is $E$, we may assume that $E$ is regular (see Proposition 2.3 or Theorem 1.12 of [6]). Let $E$ be defined by $Y$ (see [6] p. 109) and let $Y^{\prime}$ be the center of $\left(e_{Y}^{0}\right)^{-1}$. Assume that $E$ is not stable, then there is a torsion free coherent $\mathcal{O}_{X}$-module $L$ of rank 1 such that $L$ is a quotient module of $E$ and that $d(E, H) \geq 2 d(L, H)$. Then the surjective homomorphism $E \rightarrow L$ 
provides a closed subscheme $Z$ of $\mathbf{P}(E)$. Since we need only the inequality $d(E, H) \geq 2 d(L, H)$ in our proof below, we may assume that $Z$ is irreducible. Then $Z$ gives us a positive divisor $Z^{\prime}$ on $\mathbf{P}(E)$ such that $\mathcal{O}_{\mathbf{P}(E)}\left(Z^{\prime}\right) \cong \mathcal{O}_{\mathbf{P}(E)}(1) \otimes_{\mathcal{O P}(E)} \pi^{*}\left(\mathcal{O}_{X}(D)\right)$ with some divisor $D$ on $X$, where $\mathcal{O}_{\mathbf{P}(E)}(1)$ is the tautological linebundle of $E$ and $\pi: \mathbf{P}(E) \rightarrow X$ is the natural projection. Now the inequality $d(E, H) \geq 2 d(L, H)$ means that $d\left(\pi_{*}\left(\left(Z^{\prime}\right.\right.\right.$ $\left.\left.-\pi^{-1}(D)\right) . \quad\left(Z^{\prime}-\pi^{-1}(D)\right), H\right) \geq 2 d\left(\pi_{*}\left(\left(Z^{\prime}-\pi^{-1}(D)\right) \cdot Z^{\prime}\right), H\right)$, that is, $d\left(\pi_{*}\left(Z^{\prime}\right.\right.$. $\left.\left.Z^{\prime}\right), H\right) \leq 0$. Let $Z_{0}$ be the proper transform of $Z^{\prime}$ by $\operatorname{elm}_{Y^{\prime}}^{0}$ and let $\pi_{0}$ be the projection of $\mathbf{P}_{X}^{1}$ to $X$. Since $Z_{0}$ is a positive divisor and since Pic $(X) \cong Z$, we have that $Z_{0} \sim(0) \times X+\pi^{-1}\left(H_{m}\right)$ with some positive integer $m$ and $H_{m} \in\left|H^{\otimes m}\right|$. If $Z^{\prime} \supset Y^{\prime}$, then $\left(\pi_{0}\right)_{*}\left(Z_{0} \cdot Z_{0}\right)=\pi_{*}(Z \cdot Z)$, whence $d\left(\left(\pi_{0}\right)_{*}\left(Z_{0} \cdot Z_{0}\right), H\right)=d\left(\pi_{*}(Z \cdot Z), H\right)$. But this is impossible because $d\left(\pi_{*}(Z \cdot Z), H\right)$ $\leq 0$ and $d\left(\left(\pi_{0}\right)_{*}\left(Z_{0} \cdot Z_{0}\right), H\right)=2 m d(H, H)>0$. Next assume that $Z^{\prime} \not \supset Y^{\prime}$. Then $\left(\pi_{0}\right)_{*}\left(Z_{0} \cdot Z_{0}\right)=\pi_{*}(Z \cdot Z)+T$, where $T=\pi_{0}(Y)$ and $Y$ is a section of $\mathbf{P}_{T}^{1}$. If $T$ is contained in $\left|H^{\otimes r}\right|$, then the inequality $d\left(\pi_{*}(Z \cdot Z), H\right) \leq 0$ implies that $2 m \leq r$. By this and the fact that $Z_{0} \supset Y$ we know that $E$ is not simple ([6] Theorem 3.10 or p. 128 (4)).

q.e.d.

Remark A.2. 1) Let $X$ be a non-singular projective variety over $k$ with $\operatorname{Pic}(X) \cong Z$ as an abstract group and let $H$ be an ample linebundle which is a generator of Pic $(X)$. If for all $n \geq n_{0}, H^{0}\left(X, H^{\otimes n}\right) \neq 0$, then every simple vector bundle of rank 2 is of type $n_{0} d\left(H, H^{\prime}\right)-\varepsilon$ $\left(0<\forall_{\varepsilon}<\frac{1}{2}\right)$ with respect to a very ample linebundle $H^{\prime}$ on $X$.

2) Proposition A.1 does not hold unless rank of $E$ is 2 . In fact we can show the following: For every non-singular projective surface $X$, there exists a simple but not stable vector bundle of rank 3 on $X$.

II. Let $X$ be a non-singular variety over $k$ and let $(Y, T)$ be a pair of subvarieties of a $\mathbf{P}^{1}$-bundle $\pi: \mathbf{P} \rightarrow X$ and $X$ respectively. Assume that $(Y, T)$ satisfies the condition $\left(E_{0}\right)$ ([6] p. 105). Put $\mathbf{P}^{\prime}=\operatorname{elm}_{Y}^{0}(\mathbf{P})$ and let $\pi^{\prime}$ be the projection of $\mathbf{P}^{\prime}$ to $X$. Let $Y^{\prime}$ be the center of $\left(\operatorname{elm}_{Y}^{0}\right)^{-1}$. Assume that $Z$ is a positive divisor on $\mathbf{P}$ such that $\mathcal{O}_{\mathbf{P}}(Z) \cong \mathcal{O}_{\mathbf{P}}(2) \otimes_{0 \mathbf{P}} \pi^{*}(L)$ with some tautological linebundle $\mathcal{O}_{\mathbf{P}}(1)$ on $\mathbf{P}$ and some linebundle $L$ on $X$ and that $Z \not \supset \pi^{-1}(T)$. Let $Z^{\prime}$ (or, $Z^{\prime \prime}$ ) be the proper (or, total, resp.) transform of $Z$ by $\operatorname{elm}_{Y}^{0}$. Set $n=\max \left\{r \mid Z \cdot \pi^{-1}(T) \supset r Y\right\}, n^{\prime}=\max \left\{r^{\prime} \mid Z^{\prime} \cdot \pi^{\prime-1}(T)\right.$ $\left.\supset r^{\prime} Y^{\prime}\right\}$ and $m=\max \left\{s \mid Z^{\prime \prime} \supset s \pi^{\prime-1}(T)\right\}$. Then we get the following table; 


\begin{tabular}{|c|c|c|c|}
\hline \multicolumn{2}{|c|}{} & $n^{\prime}$ & $m$ \\
\hline 0 & & 2 & 0 \\
\hline 1 & & 1 & 1 \\
\hline \multirow{2}{*}{2} & Sing $(Z) \supset Y$ & 0 & 2 \\
\cline { 2 - 4 } & Sing $(Z) \not \supset Y$ & 2 & 1 \\
\hline
\end{tabular}

where Sing $(Z)$ denotes the singular locus of $Z$.

Proof. Since the problem is a local property with respect to $X$, we may assume that $X=\operatorname{Spec}(A), \quad \mathbf{P}=\operatorname{Proj}\left(A\left[\lambda_{0}, \lambda_{1}\right]\right)$ and that the defining ideal $I_{T}$ for $T$ in $A$ (or, $I_{Y}$ for $Y$ in $A\left[\lambda_{0}, \lambda_{1}\right]$ ) is generated by $t \in A$ (or, $t, \lambda_{1}$, resp.). Then $\mathbf{P}^{\prime}=\operatorname{Proj}\left(A\left[\lambda_{0}^{\prime}, \lambda_{1}^{\prime}\right]\right)$ with $\lambda_{0}=\lambda_{0}^{\prime}, \lambda_{1}=t \lambda_{1}^{\prime}$ and the defining ideal $I_{Y^{\prime}}$ for $Y^{\prime}$ in $A\left[\lambda_{0}^{\prime}, \lambda_{1}^{\prime}\right]$ is generated by $t$, $\lambda_{0}^{\prime}$ (see [6] Lemma 1.4). Shrinking $X$ if necessary, we may assume that $Z$ is defined by $a_{0} \lambda_{0}^{2}+a_{1} \lambda_{0} \lambda_{1}+a_{2} \lambda_{1}^{2}=0$ with $\left(a_{0}, a_{1}, a_{2}\right) A \not \subset t A$.

1) Assume that $a_{0} A \not \subset t A$, then $Z^{\prime}=Z^{\prime \prime}$ and they are defined by $a_{0} \lambda_{0}^{\prime 2}+a_{1} t \lambda_{0}^{\prime} \lambda_{1}^{\prime}+a_{2} t^{2} \lambda_{1}^{\prime 2}=0$. Since $Z \cdot \pi^{-1}(T)$ (or, $Z^{\prime} \cdot \pi^{\prime-1}(T)$ ) is defined by $\bar{a}_{0} \bar{\lambda}_{0}^{2}+\bar{a}_{1} \bar{\lambda}_{0} \bar{\lambda}_{1}+\bar{a}_{2} \bar{\lambda}_{1}^{2}=0$ (or, $\bar{a}_{0} \bar{\lambda}_{0}^{\prime 2}=0$, resp.), we have that $n=0, n^{\prime}=2$, $m=0$, where - denotes the homomorphic image by the natural homomorphism of $A\left[\lambda_{0}, \lambda_{1}\right]$ (or, $A\left[\lambda_{0}^{\prime}, \lambda_{1}^{\prime}\right]$ ) to the homogeneous ring $(A / t A)\left[\bar{\lambda}_{0}, \bar{\lambda}_{1}\right]$ of $\pi^{-1}(T)$ (or, $(A / t A)\left[\bar{\lambda}_{0}^{\prime}, \bar{\lambda}_{1}^{\prime}\right]$ of $\pi^{\prime-1}(T)$, resp.).

2) Assume that $a_{0} A \subset t A, a_{1} A \not \subset t A$, then $Z^{\prime}$ (or, $Z^{\prime \prime}$ ) is defined by $a_{0}^{\prime} \lambda_{0}^{\prime 2}+a_{1} \lambda_{0}^{\prime} \lambda_{1}^{\prime}+a_{2} t \lambda_{1}^{\prime 2}=0$ (or, $a_{0}^{\prime} t \lambda_{0}^{\prime 2}+a_{1} t \lambda_{0}^{\prime} \lambda_{1}^{\prime}+a_{2} t^{2} \lambda_{1}^{\prime 2}=0$, resp.), where $a_{0}=$ $a_{0}^{\prime} t$. Since $Z \cdot \pi^{-1}(T)$ (or, $Z^{\prime} \cdot \pi^{\prime-1}(T)$ is defined by $\left(\bar{a}_{1} \bar{\lambda}_{0}+\bar{a}_{2} \bar{\lambda}_{1}\right) \bar{\lambda}_{1}=0$ (or, $\left(\bar{a}_{0}^{\prime} \bar{\lambda}_{0}^{\prime}+\right.$ $\left.\bar{a}_{1} \bar{\lambda}_{1}^{\prime}\right) \bar{\lambda}_{0}^{\prime}=0$, resp.) and since $\bar{a}_{1} \neq 0$, we have that $n=1, n^{\prime}=1$ and $m=1$.

3) Assume that $a_{0} A \subset t A, a_{0} A \not \subset t^{2} A$ and $a_{1} A \subset t A$. Then by the assumption that $Z \not \supset \pi^{-1}(T)$, we know that $a_{2} A \not \subset t A$. In this case $Z^{\prime}$ (or, $Z^{\prime \prime}$ ) is defined by $a_{0}^{\prime} \lambda_{0}^{\prime 2}+a_{1}^{\prime} t \lambda_{0}^{\prime} \lambda_{1}^{\prime}+a_{2} t \lambda_{1}^{\prime 2}=0$ (or, $a_{0}^{\prime} t \lambda_{0}^{\prime 2}+a_{1}^{\prime} t^{2} \lambda_{0}^{\prime} \lambda_{1}^{\prime}+a_{2} t^{2} \lambda_{1}^{\prime 2}$ $=0$, resp.), where $a_{0}=a_{0}^{\prime} t, a_{1}=a_{1}^{\prime} t$. Since $Z \cdot \pi^{-1}(T)$ (or, $Z^{\prime} \cdot \pi^{\prime-1}(T)$ ) is defined by $\bar{a}_{2} \bar{\lambda}_{1}^{2}=0$ (or, $\bar{a}_{0}^{\prime} \bar{\lambda}_{0}^{\prime 2}=0$, resp.) and since $\bar{a}_{2} \neq 0, \bar{a}_{0}^{\prime} \neq 0$, we have that $n=2, n^{\prime}=2$ and $m=1$. Moreover if $y$ is the generic point of $Y$, then $\mathcal{O}_{\mathbf{P}, y}$ is a regular local ring whose maximal ideal $\mathfrak{m}_{y}$ is generated by $t, \lambda=\lambda_{1} / \lambda_{0}$ and the defining ideal for $Z$ at $y$ is generated by $z_{1}=a_{0}^{\prime} t$ $+a_{1}^{\prime} t \lambda+a_{2} \lambda^{2}$. Since $a_{0}^{\prime}$ is a unit in $\mathcal{O}_{\mathbf{P}, y}, z_{1}$ is contained in $\mathfrak{m}_{y}$ but not in $\mathfrak{m}_{y}^{2}$, whence $Z$ is simple at $y$.

4) Assume that $a_{0} A \subset t^{2} A$ and $a_{1} A \subset t A$. In this case we know also that $a_{2} A \not \subset t A$. Then $Z^{\prime}$ (or, $Z^{\prime \prime}$ ) is defined by $a_{0}^{\prime \prime} \lambda_{0}^{\prime 2}+a_{1}^{\prime} \lambda_{0}^{\prime} \lambda_{1}^{\prime}+a_{2} \lambda_{1}^{\prime 2}$ 
$=0$ (or, $a_{0}^{\prime \prime} t^{2} \lambda_{0}^{\prime 2}+a_{1}^{\prime} t^{2} \lambda_{0}^{\prime} \lambda_{1}^{\prime}+a_{2} t^{2} \lambda_{1}^{\prime 2}=0$, resp.), where $a_{0}=a_{0}^{\prime \prime} t^{2}, a_{1}=a_{1}^{\prime} t$. Since $Z \cdot \pi^{-1}(T)$ (or, $Z^{\prime} \cdot \pi^{\prime-1}(T)$ ) is defined by $\bar{a}_{2} \bar{\lambda}_{1}^{2}=0$ (or, $\bar{a}_{0}^{\prime \prime} \bar{\lambda}_{0}^{\prime 2}+\bar{a}_{1}^{\prime} \bar{\lambda}_{0}^{\prime} \bar{\lambda}_{1}^{\prime}$ $+\bar{a}_{2} \bar{\lambda}_{1}^{\prime 2}=0$, resp.), we have that $n=2, n^{\prime}=0$ and $m=2$. Moreover the defining ideal for $Z$ at the generic point $y$ of $Y$ is generated by $z_{2}=a_{0}^{\prime} t^{2}+a_{1}^{\prime} t \lambda+a_{2} \lambda^{2}$ and $z_{2} \in \mathfrak{m}_{y}^{2}$. Thus $y$ is a singular point of $Z$.

Combining all the above results we get the desired table. q.e.d.

III. To show that the main theorem of $\S 4$ is not trivial let us prove the following proposition.

Proposition A.3. Let $X$ be a non-singular projective surface over $k$ and let $H$ be a very ample linebundle on $X$. For a given divisor $D$ on $X$, there exists an integer $n_{0}$ such that for any $n \geq n_{0}$, there exists a stable vector bundle $E$ of rank 2 on $X$ with respect to $H$ with $c_{1}(E)$ $=$ the class of $D$ and $c_{2}(E)=n$.

Proof. Replacing $D$ by $D+H_{m}$ with a suitable $H_{m}$ in $\left|H^{\otimes m}\right|$, we may assume that $|D|$ contains a non-singular irreducible curve. Pick a non-singular irreducible member $T$ in $|D|$. Let $\mathscr{A}$ be the set of positive divisors $C$ with $d(T, H) / 2 \geq d(C, H)$ and put $\mathscr{N}=\mathscr{A} /$ (numerical equivalence). Then $\mathscr{N}$ is a finite set. Set $n_{1}=\max \{(T, C) \mid C \in \mathscr{A}\}+1$. Since $\mathscr{N}$ is a finite set and since $(T, C)$ depends only on the numerical equivalence class of $C, n_{1}$ is finite. Now let us show that $n_{0}=\max \left\{n_{1}\right.$, genus of $\left.T\right\}$ is one of the desired integers. Take an integer $n \geq n_{0}$, then there exists a divisor $B$ on $T$ such that $\operatorname{deg} B=n$ and $|B|$ is free from base points because $n \geq$ genus of $T$. Then $R^{2}(X, T, B)$ is not empty (see [6] p. 112). Let us take a member $E$ of $R^{2}(X, T, B)$ which is defined by $Y$. Since $c_{1}(E)=$ the class of $D$ and $c_{2}(E)=\operatorname{deg} B=n$, we have only to prove that $E$ is stable. Assume that $E$ is not stable and take a torsion free coherent $\mathcal{O}_{X}$-module $L$ and divisors $Z^{\prime}, Z_{0}$ on $\mathbf{P}(E), \mathbf{P}_{X}^{1}$ respectively as in the proof of Proposition A.1. If $Z_{0}$ does not contain $Y$, then $d(L, H)$ $\geq d(T, H)>d(E, H) / 2$. Thus $Z_{0}$ has to contain $Y$. Moreover $Z_{0} \sim(0) \times X$ $+\pi_{0}^{-1}(C)$ with some positive divisor $C$ on $X$. The inequality $d(E, H) / 2$ $\geq d(L, H)$ implies that $d(T, H) / 2 \geq d(C, H)$. On the other hand, since (0) $\times T+\left(\pi_{0}\right)_{T}^{-1}(C \cdot T) \sim Z_{0} \cdot \pi_{0}^{-1}(T) \sim Y+\left(\pi_{0}\right)_{T}^{-1}\left(B^{\prime}\right)$ with some positive divisor $B^{\prime}$, we get that $(T, C)=\operatorname{deg}\left(B+B^{\prime}\right) \geq n \geq n_{0}$. This is impossible because $C$ is contained in $\mathscr{A}$. Thus $E$ is stable. q.e.d. 


\section{REFERENCES}

[1 ] A. Borel and J.-P. Serre, Le théorème de Riemann-Roch. Bull. Soc. Math. France, 86 (1958).

[2] A. Grothendieck, Techniques de construction et théorèmes d'existence en géométrie algébrique IV: Les schémas de Hilbert, Séminaire Bourbaki, t. 13, 1960/61, $n^{\circ}$ 221.

[E.G.A.] A. Grothendieck and J. Dieudonné, Éléments de Géométrie Algébrique, Chaps. I, II, III, IV, Publ. Math. I. H. E. S., Nos. 4, 8, 11, 17, 20, 24, 28 and 32.

[3] G. Horrocks, Vector bundles on the punctured spectrum of a local ring, Proc. London Math. Soc. (3) 14 (1964).

[4] S. Kleiman, Les théorèmes de finitude pour le foncteur de Picard. Séminair de Géométrie Algébrique du Bois Marie, 1966/67 (S.G.A.6), Expose XIII, Lecture Notes in Math., 225, Springer-Verlag, Berlin-Heidelberg-New York (1971).

[5] D. Knutson, Algebraic Spaces, Lecture Notes in Math., 203, Springer-Verlag, Berlin-Heidelberg-New York (1971).

[6 ] M. Maruyama, On a family of algebraic vector bundles, Number Theory, Algebraic Geometry and Commutative Algebra, in honor of Y. Akizuki, Kinokuniya, Tokyo (1973).

[ 7 ] D. Mumford, Projective invariants of projective structures and applications, Proc. Intern. Cong. Math., Stockholm (1962).

[8] D. Mumford, Geometric Invariant Theory, Springer-Verlag, Berlin-HeidelbergNew York (1965).

[9] D. Mumford, Lectures on Curves on an Algebraic Surface, Annals of Math. Studies, No. 59, Princeton Univ. Press (1966).

[10] D. Mumford, Abelian Varieties, Oxford Univ. Press, Bombay (1970).

[11] M. S. Narasimhan and C. S. Seshadri, Stable and unitary vector bundles on a compact Riemann surface, Ann. of Math. (2) 82 (1965).

[12] C. S. Seshadri, Space of unitary vector bundles on a compact Riemann surface, Ann. of Math. (2) 85 (1967).

[13] C. S. Seshadri, Mumford's conjecture for GL(2) and applications, Proc. Bombay Colloq. on Algebraic Geometry, Oxford Univ. Press, Bombay (1969).

[14] C. S. Seshadri, Quotient spaces modulo reductive algebraic groups, Ann. of Math. (2) 95 (1972).

[15] F. Takemoto, Stable vector bundles on algebraic surfaces, Nagoya Math. Jour. 47 (1972).

Department of Mathematics

Kyoto University 